\title{
The University of Virginia Experimental and Theoretical High Energy Physics Final Closeout Report
}

\author{
August 13, 2012 \\ University of Virginia \\ Charlottesville, VA \\ DoE Grant Closeout Report
}

DOE Contract Number: DE-FG02-97ER41027

Principal Investigator:

Harry B. Thacker

UVA Physics Department

P.O. Box 400714

Charlottesville, VA 22904-4714

e-mail: hbt8r@virginia.edu

phone: (434)924-3204

After commitments through April, 2012, there are no unexpended funds in the budget. 


\section{Task A: Introduction}

The Theory group supported by Task A includes three tenured faculty, Peter Arnold, P. Q. Hung, and Hank Thacker, and one tenure-track assistant professor, Diana Vaman, who joined the UVa faculty in the Fall of 2007 and was added to task A in 2010 .

In addition to the four faculty members, Task A supports a postdoctoral position, currently held by Philip Szepietowski, as well as partial support for several graduate students. 


\section{P. Arnold and D. Vaman (joint work)}

Over the last three years, one of the major thrusts of both P. Arnold and D. Vaman's research has been a joint project on using gauge-gravity duality to discover qualitatively new results on jet quenching in strongly-coupled QCD-like plasmas. Our joint work also covers some spin-offs, made possible by the technical methods we developed, related to 2nd-order hydrodynamics of such plasmas. This section of the proposal discusses our joint work; our separate, independent projects will be covered in later sections.

We will try to keep our overview of past work relatively broad and hopefully accessible. Readers desiring more detailed discussion of the main results may find useful sections I and II of ref. [AV2] and section I of ref. [AV3]. (See the publication list on p. 9 for references to our own recent work, labeled here as AV1, AV2, ... to indicate Arnold-Vaman-1, etc.)

Though our work was originally motivated by a particular application, the work produced general theoretical progress on applying gauge-gravity duality to real-time finite-temperature problems. Specifically, we have developed a simple practical procedure for directly computing 3-point (and higher-point) real-time finite-temperature correlation functions with gauge-gravity duality. Previously, there was a great deal of work in the literature on how to compute 2-point real-time correlators at finite temperature, used to find viscosity and many other properties of stronglycoupled QCD-like plasmas with gravity duals, but it was unclear how to practically implement any of the suggestions in the literature relevant to 3-point correlators. (We will explain below what 3-point correlators have to do with jet quenching and other applications.)

\section{Jet Quenching}

In relativistic heavy ion collisions, experimentalists and phenomenologists use high-energy jets as an important probe of the properties of the QCD fireball left behind by the collision. The energy lost by away-side jets drilling through the fireball offers a handle on the characteristics of the fireball. This motivates various theoretical problems in how to calculate such effects from first principles in QCD. One of the most basic such problems is the following: How far will a very high energy parton (or collection of partons it evolves into) travel in a quark-gluon plasma before effectively stopping and thermalizing? High energy here means that the parton's energy $E$ is large compared to the quark-gluon plasma's temperature $T$.

For the sake of simplifying the presentation, we will focus on an even more basic question: How does this stopping distance scale with the energy $E$ of the initial high-energy parton? This question alone showcases a lot of interesting physics.

Before continuing, we should make one clarification. Having uttered the phrase "relativistic heavy ion collisions" above, we should emphasize that our primary motivation has been to make progress in understanding the rich theoretical structure of all gauge theories at finite temperature. Our work is of a general nature concerning the properties and dynamics of thermal gauge theories, whether they be quark-gluon plasmas today or quark-gluon, electroweak, technicolor, or other plasmas in the very early universe. As high-energy theorists, our emphasis is squarely on fundamental, first-principles results in quantum field theory and beyond.

\section{Background}

Consider first the limit of a weakly-coupled quark-gluon plasma, which is the formal limit where one assumes the temperature is so high that the running coupling $\alpha_{\mathrm{s}}(T)$ can be treated as small. In that case, high-energy partons lose energy primarily by hard, nearly-collinear bremsstrahlung or 


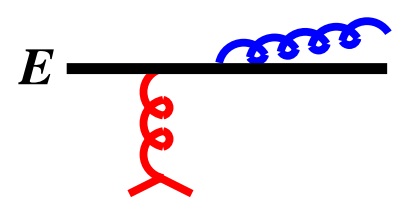

(a)

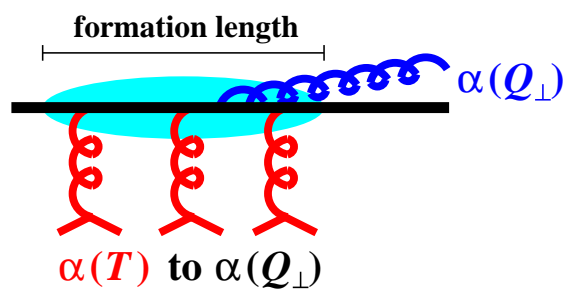

(b)

Figure 1: (a) Matrix element for a naive calculation of the bremsstrahlung rate. (b) LPM effect requires accounting for multiple collisions during the formation of the bremsstrahlung gauge boson.

pair production. Repeated splitting develops a shower akin to a cosmic ray shower or the shower of a particle in a calorimeter. A naive calculation of the rate for such splitting, based on diagrams like Fig. 1a, predicts that the rate for high-energy bremsstrahlung is energy independent (up to logarithms). At very high energy, however, the internal straight line propagator in Fig. 1a becomes so close to being on-shell that the quantum mechanical duration of this process, known as the formation time, exceeds the mean free time between collisions. At such energies, each collision with the medium does not individually provide an independent opportunity for bremsstrahlung, and the bremsstrahlung rate is correspondingly reduced. This suppression is known as the LandauPomeranchuk-Migdal (LPM) effect. The result turns out to be that the rate for hard bremsstrahlung shrinks with energy as $E^{-1 / 2}$ (up to logarithms) at high energy, and correspondingly the stopping distance for high energy partons grows as $E^{1 / 2}$.

To calculate this effect in weak coupling, one must self-consistently incorporate all of the collisions during the formation time, as depicted qualitatively in Fig. 1b for the QED case. (For QCD, the brem gluon interacts with the medium as well during its formation.)

What sets the scale for the running coupling constant in this process? As the particle flies along, it picks up small kicks from the medium, which change its direction. Changes in direction are a form of acceleration, and so a source for bremsstrahlung radiation. The individual kicks are dominated by relatively soft momentum scales for which $\alpha_{\mathrm{s}}$ is of order $\alpha_{\mathrm{s}}(T)$. But now consider the $\alpha_{\mathrm{s}}$ associated with the emission of the bremsstrahlung gluon. Its scale is set by the relative transverse momenta $Q_{\perp}$ of the two daughter particles after the splitting. In an infinite medium (the relevant case if the medium is thick enough for a jet to actually stop), the typical $Q_{\perp}$ turns out to grow slowly with particle energy as $Q_{\perp} \sim(\hat{q} E)^{1 / 4}$. Here $\hat{q}$ is a characteristic of the medium which quantifies the net squared transverse momentum picked up per unit length that a high-energy particle travels through the medium.

The scaling of the stopping distance $\ell_{\text {stop }}$ depends on the size of $\alpha_{\mathrm{s}}$ at these two scales $T$ and $Q_{\perp}$.

- weak coupling: If $\alpha_{\mathrm{S}}(T) \sim \alpha_{\mathrm{S}}\left(Q_{\perp}\right)$ are both small, then it is known that $\ell_{\text {stop }} \propto E^{1 / 2}$ (up to logarithms), as discussed earlier.

- mixed coupling: If $\alpha_{\mathrm{s}}(T)$ is large and $\alpha_{\mathrm{s}}\left(Q_{\perp}\right)$ is small, various people have argued that the stopping distance still scales as $\ell_{\text {stop }} \propto E^{1 / 2}$ and that the strong-coupling dynamics of the problem can be isolated into the value of $\hat{q}$. This has previously motivated exploration of the value of $\hat{q}$ in strongly-coupled QCD-like theories using gauge-gravity duality [1].

- all strong coupling: For $\alpha_{\mathrm{s}}(T)=\alpha_{\mathrm{s}}\left(Q_{\perp}\right)$ very large, progress has been made in theories such 


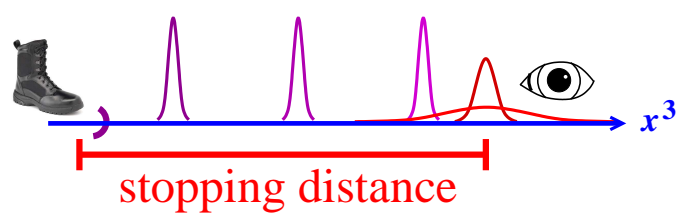

Figure 2: Time-evolution due to a high-energy kick to the plasma, with redder curves corresponding to the charge distribution at later times.
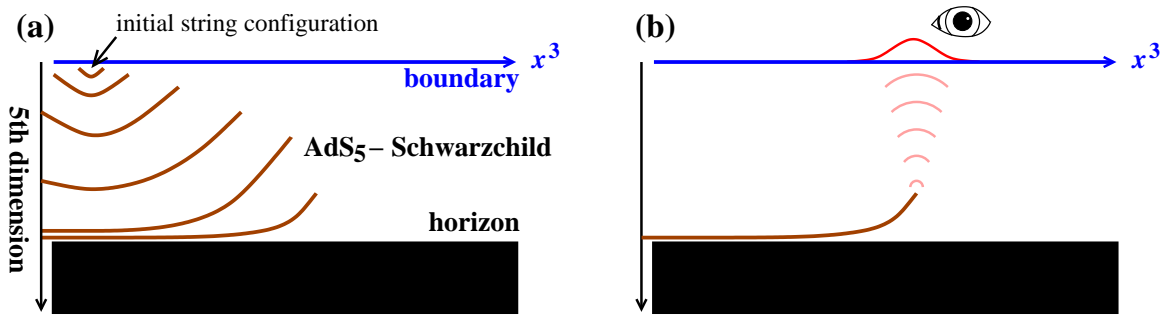

Figure 3: Cartoon of (a) the falling string and (b) its effect on the boundary.

as $\mathcal{N}=4$ super Yang Mills (and its close cousins), where calculations are possible using gaugegravity duality. In this case, people $[2,3,4]$ have found that the stopping distance has a different scaling with energy: $\ell_{\text {stop }} \propto E^{1 / 3}$.

In our work together, we have focused on further study of the last, all strong-coupling case. What is interesting about the $E^{1 / 3}$ is its demonstration that the exponent $\nu$ in $\ell_{\text {stop }} \propto E^{\nu}$ can depend on $\alpha_{\mathrm{s}}\left(Q_{\perp}\right)$. As we will discuss, we have discovered an even richer set of exponents than $\ell_{\text {stop }} \propto E^{1 / 3}$.

Think about the finite-temperature $3+1$ dimensional field theory and what happens if you very energetically kick the medium in some way at $t=0$, creating a localized excitation that moves through the medium, as depicted in fig. 2. At later times, look at the progress of the excitation through the medium by following some conserved density such as energy or momentum or charge density. For this discussion, it will be simplest if we think of a charge density. As time passes, the charge density moves in a pulse that eventually slows (because of energy loss) and then stops as the excitation thermalizes, after which the charge diffuses outward. The distance from the kick to the center of the late-time diffusion cloud of charge is the stopping distance.

As an example of previous calculations of jet stopping in AdS/CFT which found $\ell_{\text {stop }} \propto E^{1 / 3}$, fig. 3 reviews how Chesler et al. [4] set up the problem for light-quark jets. A classical string in the 5-dimensional gravity theory is used to describe a state analogous to a $q \bar{q}$ pair in QCD. The string is initialized in a way that its end points fly apart, analogous to a high-energy back-to-back quark and anti-quark. As the ends fly apart, the string falls in the fifth dimension towards the black brane horizon, as depicted by the time-series of curves in fig. 3a. The string disturbs the background gravitational and other fields, and so causes a deformation of the fields on the boundary, as depicted in fig. 3b. As in fig. 2, it will be pictorially simplest to discuss observing conserved charge densities which diffuse. As the string falls toward the horizon, its effect on the boundary becomes more and more red-shifted, and so spreads out to longer and longer wavelengths. This is how the falling string produces diffusion of the charge density seen in the boundary theory.

There is one slightly dissatisfying aspect of this very nice picture: The initial configuration of the system is specified in the language of the gravity dual rather than directly in the $3+1$ 


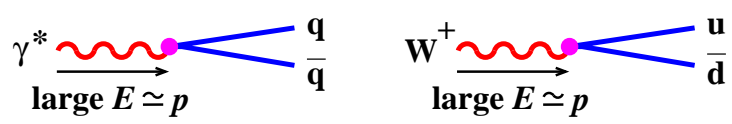

Figure 4: Examples of decays creating QCD jets.

$$
\begin{aligned}
\mid \text { jet }\rangle & =\mathbb{\text { plasma } \rangle} \\
\langle\text { jet }| \text { - jet }\rangle & =\left\langle\mathbb{S}^{\dagger} \circlearrowleft\right\rangle
\end{aligned}
$$

Figure 5: Relation of observable (eyeball) to 3-point correlator.

dimensional field theory. Our goal was to avoid this by formulating some "jet" stopping problem from beginning to end in the field theory. We only use the gravity dual as a means to solve that field-theory problem.

\section{Our Method}

To understand our method, first think impessionistically of the decay of a very high energy, slightly virtual photon, or of a very high energy $W^{+}$boson, in a QCD quark-gluon plasma. Fig. 4 shows the corresponding processes for weak coupling. Each creates a localized, high-energy, high-momentum excitation in the plasma, moving towards the right of the figure. We will loosely call such an excitation a "jet."

Now treat the red photon or $W$ line in fig. 4 as an external field in the form of a high-energy plane wave $e^{i \bar{k} \cdot x}$ with $\bar{k}^{\mu}=(E, 0,0, E)$. But we will also approximately localize that external field, so that we know where the jet it creates starts from. When we see where the jet stops, we may then extract the stopping distance from the difference. So we replace the photon or $W$ by adding a source term to the Lagrangian:

$$
\mathcal{L}_{\mathrm{QFT}} \rightarrow \mathcal{L}_{\mathrm{QFT}}+\mathcal{O}(x) \Lambda_{L}(x) e^{i \bar{k} \cdot x}
$$

The factor $\Lambda_{L}(x) e^{i \bar{k} \cdot x}$ is the external field, where $\Lambda_{L}(x)$ is a smooth envelope function which localizes the source to a region of size $L$ around the origin in both space and time. The $\mathcal{O}(x)$ is the field-theory operator the external field couples to, such as $j_{\mu}(x)$ in the examples of fig. 4 , and corresponds to the vertex in fig. 4. (For referees interested in a discussion of the similarities and differences of this set up with the zero-temperature work of Hofman and Maldacena, see section I.C of ref. $[\mathrm{AV} 2]$.

It turns out that the response to such a source is measured by a 3-point correlator in the field theory. A crude but simple way to understand this is given in fig. 5. The boot represents the source operator in (1). We apply this operator to a generic state of the thermal plasma to create the localized, high-energy "jet" state, as in fig. 5a. We then want the expectation value in this state of some conserved density - energy or momentum or charge - represented by the eyeball operator in fig. 5b. Substituting fig. 5a into the left-hand side of fig. 5b yields the right-hand side of fig. $5 \mathrm{~b}$, which is a 3-point correlator of the boot, the eyeball, and the boot-conjugate, calculated for an equilibrium plasma. For finite-temperature AdS/CFT calculations, there is a great deal in the literature on computing 2-point correlators, but until recently almost nothing carried through to specific results for 3 -point correlators. 


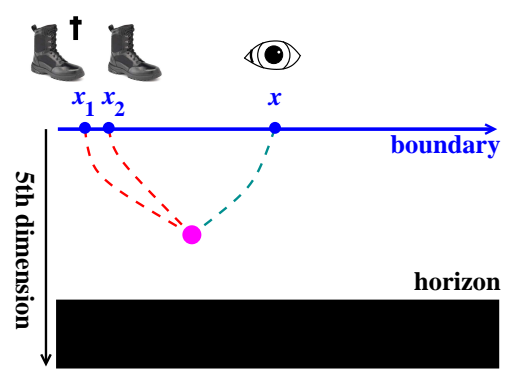

Figure 6: Tree diagram giving the 3-point correlator in the gravity dual theory.

To compute this field-theory three-point correlator, we use gauge-gravity duality to relate it to a three-point boundary correlator in $\mathrm{AdS}_{5}$-Schwarzschild space, as depicted in fig. 6 . Here $x$ is the space-time point where we make our observation, and $x_{1}$ and $x_{2}$ are integrated over the source region. Recall that gauge-gravity duality relates the strongly-coupled field theory problem to a problem in classical gravity, which means tree diagrams in the gravity problem. In Fig. 6, we therefore have a three-leaf tree diagram made up of the dashed lines meeting at a vertex in the bulk. The vertex represents a cubic term in the 5-dimensional supergravity action. The dashed lines are bulk-to-boundary propagators.

\section{3-point correlators}

For generic real-time 3-point correlators at finite temperature, there is an apparent difficulty when integrating over the location of the bulk vertex in Fig. 6: namely, the bulk vertex could be behind the horizon (or in any of the quadrants of the Penrose diagram) or even at the singularity. There have been various proposals for making sense of this situation, including one to complexify the space-time behind the horizon in analogy to the complex-time path-integral contours used in realtime finite-temperature field theory. But prior to our work [AV1], there was no straightforward practical-to-implement prescription for computing 3-point functions. Together with postdoc Eddy Barnes and graduate student Chaolun $\mathrm{Wu}$, we showed how to write formulas for most types of correlators in a way that only involves integrating over regions of the Penrose diagram outside of the horizon.

Especially simple is the retarded 3-point correlator. In ref. [AV2], we showed how to formulate the jet stopping problem in terms of retarded correlators, for which the bulk vertex in fig. 6 has to remain above the horizon simply because there is no way for a causal signal to propagate from the source to below the horizon and then return to influence the measurement point on the boundary. We showed how integral expressions for the 3-point correlator in imaginary-time (where the geometry is smooth and there are no singularities to worry about) can be analytically continued to give appropriate expressions for the retarded 3-point correlator in real time. (See sections I.B and I.C.1 of ref. [AV2] if interested in more detail.)

\section{Calculating the 3-point correlator}

The bulk-to-boundary propagators in fig. 6 are given by Heun functions. Heun functions are obscure generalizations of hypergeometric functions which are difficult to work with. As a result, it is difficult to make analytic progress in calculating generic 3-point functions, which requires integrating over the vertex position. It is also difficult to evaluate such integrals numerically in 


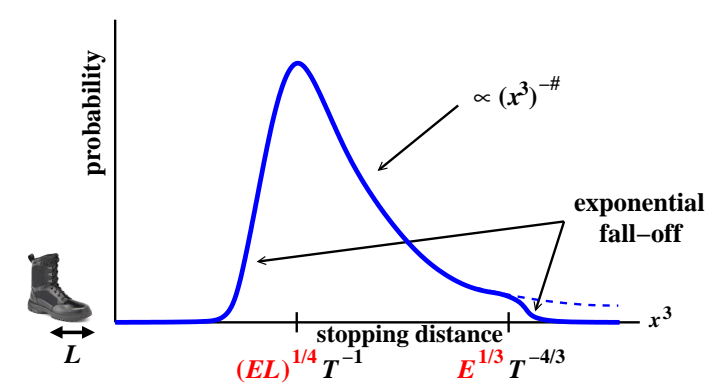

Figure 7: Qualitative picture of the probability distribution of jet stopping distances. (The dashed curve shows the 5-dimensional "particle" approximation where it is different from the exact result.)

our problem because the fact that our source is high energy, and the fact that we are looking at real-time correlations, means that the bulk-to-boundary propagators associated with the boots are very highly oscillatory functions and so very difficult to integrate.

Fortunately, this very problem also points the way to a solution. The (red) dashed lines in fig. 6 associated with the boots are high-energy/momentum propagators, and we may make a corresponding high- $k$ WKB-like approximation, which gives them a relatively simple analytic form. For the remaining (green) dashed line in fig. 6, recall that the technique for measuring the stopping distance is to look for the spatial center of late-time charge diffusion, as depicted by the last curve in Fig. 2. This corresponds to measurements of large wavelengths and so small wave numbers of the late-time response of the system. As a result, we may use a low- $k$ approximation to the (green) dashed line associated with the eyeball in Fig. 6. The low- $k$ limit also gives a relatively simple analytic expression for the propagator. These approximations, appropriate for the problem we want to solve, turn out to be enough to make it possible to analytically integrate over the bulk vertex position and compute the 3-point correlator.

The results [AV2,AV3] of using this method to calculate the jet stopping distance are summarized in Fig. 7, which shows a probability distribution for the jet stopping distance. There is indeed a "maximum" stopping distance that scales like $E^{1 / 3}$, beyond which the probability distribution falls off exponentially. However, this is not the typical stopping distance of the jets created. Almost all of the jets created by our kick (1) instead stop sooner at a distance scale proportional to $(E L)^{1 / 4}$, where $L$ is the size of the space-time region in which the jet was initially created (i.e. the size of the toe of our boot and the duration of the kick). Between the scales $(E L)^{1 / 4}$ and $E^{1 / 3}$, the probability distribution falls algebraically.

\section{5-dimensional particles}

Why should the source size $L$ affect the typical stopping distance? One possibility is that the source size determines the typical virtuality $q^{2}$ of the jets. That's because a finite source size means that the components $q^{\mu}$ of the sources 4 -momentum each have a spread of order $1 / L$ around the value $\bar{k}^{\mu}=(E, 0,0, E)$ used in the source (1), depicted in fig. 8a. This introduces a virtuality of order $\left|q^{2}\right| \sim E / L$. In order to check this, we replaced $\bar{k}^{\mu}$ by $(E+\delta, 0,0, E-\delta)$ with $L^{-1} \ll \delta \ll E$, as depicted in fig. 8b. This makes the virtuality of the source $q^{2} \sim-\delta E$, and so one may test whether the typical stopping distance is sensitive to virtuality $(\delta)$ or just the source size $(L)$. We found that the 5-dimensional excitation created by this type of source (that is, the 5 -dimensional wave you get by convolving the source with a bulk-to-boundary propagator) was a very narrow, very wiggly wave packet. That is, the excitation was a wave packet whose size in the 5 th dimension was parametrically 


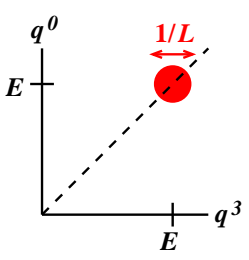

(a)

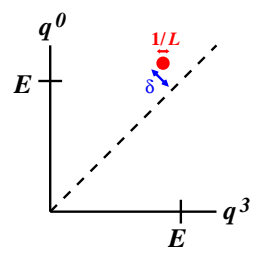

(b)

Figure 8: The support in momentum space $q$ of a localized source (1) with (a) $\bar{k}=(E, 0,0, E)$ and (b) $\bar{k}=(E+\delta, 0,0, E-\delta)$, where $L^{-1} \ll \delta \ll E$.

small compared to the scale over which the geometry changes but parametrically large compared to the wavelength oscillations. Such wave packets have both well defined momentum and position (up to parametrically small uncertainties) and so their behavior is the same as that of particles. This is just the geometric optics (or "ray tracing") limit in the theory of waves. Because we apply this approximation to 5-dimensional fields in the gravity dual, these "particles" are particles in 5 dimensions rather than 4 .

Through a very simple calculation of null geodesics corresponding to classical 5-dimensional particles in the gravity dual, ${ }^{1}$ we were able to show that the stopping distance is determined by the virtuality of the source by

$$
\ell_{\text {stop }} \sim\left(\frac{E}{\delta}\right)^{1 / 4} \sim\left(\frac{E^{2}}{q^{2}}\right)^{1 / 4}
$$

as long as this distance remains large compared to the source size itself. (The breakdown of this condition, which happens at stopping distances of order the maximum stopping distance scale $E^{1 / 3}$, is where the particle approximation breaks down.) We also showed how these simple particle results could be superposed to exactly reproduce the $\ell \ll E^{1 / 3}$ part of the earlier result of fig. 7 . As far as typical stopping distances go, this gives a quick, simple, easy-to-understand way of finding results, in place of the much more involved and laborious wave analysis originally used to obtain Fig. 7.

\section{4-point correlators}

Having demonstrated practical computations of real-time finite-temperature 3-point correlators with gauge-gravity duality, we showed how to apply the same methods to a 4-point correlator in ref. [AV5]. The particular application we studied was whether there was any correlation between jet charge deposition at different distances from the source. The original derivation of the result in fig. 7 for jet stopping distances had just been a calculation of the expectation value of the charge deposition, related to the expectation value of a single observable as shown in fig. 5b. Such a calculation does not clearly distinguish whether the spread of stopping distances in fig. 7 represents (i) a probability distribution for how far each jet goes, or (ii) a situation where each jet individually deposits charge across a variety of different distances. The first interpretation turns out to be the correct one, and we checked this by studying the correlator between making measurements of deposition at different positions:

$$
\langle\text { jet }|\circlearrowleft(x) \circlearrowleft(y)| \text { jet }\rangle=\left\langle\mathbb{S}^{\dagger} \circlearrowleft(x) \circlearrowleft(y) d\right\rangle
$$

An example of a corresponding tree diagram in the gravity dual is shown in fig. 9 .

\footnotetext{
${ }^{1}$ We were by no means the first people to work out 5 -dimensional null geodesics for various purposes in the gravity dual. But the application of our calculations (to understand our "typical" stopping distance scale) was novel.
} 


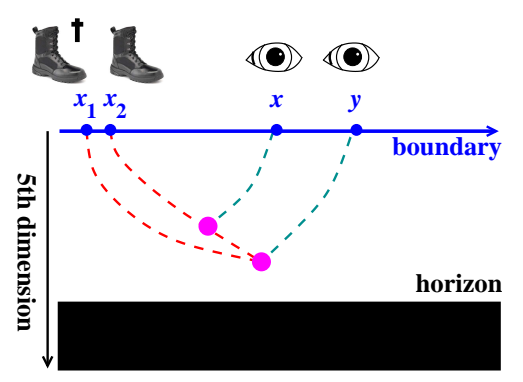

Figure 9: Example of a tree diagram giving the 4-point correlator in the gravity dual theory.

\section{2nd-order hydrodynamics}

On distance and time scales large compared to mean free paths and mean free times between collisions, a hot non-abelian plasma behaves like any other gas or fluid and obeys the equations of hydrodynamics. If one naively writes down the equations of relativistic viscous hydrodynamics, one finds instabilities and acausal behavior. There is nothing in principle wrong because these pathologies are associated with fluctuations whose wavelengths are small enough that the hydrodynamic description is breaking down. Like any effective theory, hydrodynamics mangles the physics at very short distance scales where the effective theory does not apply. That is little comfort, however, if one wishes to do numerical simulations of the hydrodynamics, since unphysical instabilities will make numerical simulation impractical. For this reason, physicists who numerically simulate relativistic viscous hydrodynamics turned to what are known as "2nd-order" hydrodynamics equations. These versions of hydrodynamics include (typically a subset of) the leading corrections to hydrodynamics in the long-distance limit. If done the right way, including such corrections also cures the short-distance pathologies of the effective theory and so allows for numerical simulation. The cost of this fix is the introduction of more parameters to the hydrodynamic description, parametrizing the size of the correction terms. Formally, the precise value of these parameters, known as 2nd-order hydrodynamic coefficients, should not matter in the long-distance limit where hydrodynamics is valid. In practice, though, if one is incorporating them into simulations, it makes sense to choose the values as close to their actual values as possible. It is possible to compute their values in the weak coupling limit $\alpha \ll 1$. It is also possible to compute them in strongly-coupled theories with gravity duals.

"1st-order" hydrodynamic coefficients, like viscosity, are related to the low-frequency lowmomentum limit of retarded correlators of conserved currents through what are known as Kubo formulas. For example, the shear viscosity $\eta$ is given in terms of retarded correlators of the stressenergy tensor by

$$
\eta=-\lim _{\omega \rightarrow 0} \lim _{\boldsymbol{k} \rightarrow 0} G_{\mathrm{R}}^{x y, x y}(\omega, \boldsymbol{k}),
$$

where $G_{\mathrm{R}}^{x y, x y}$ is the Fourier transform of $\left\langle\left[T^{x y}(x), T^{x y}(0)\right]\right\rangle \theta\left(x^{0}\right)$. This was the starting point for Policastro, Son, and Starinets' derivation of the viscosity of strongly coupled theories with gravity duals. $^{2}$ Later, Baier et al. [6] showed how to similarly relate some of the 2nd-order hydrodynamic coefficients to 2-point retarded correlators and calculate them for such theories. A different set of 2nd-order coefficients were computed by an entirely different method by Bhattacharyya et al. [7], and the union of the two group's results covered all the coefficients. Together with graduate students $\mathrm{Wu}$ and Xiao, we wanted to find a way to compute (and in the process check) all the

\footnotetext{
${ }^{2}$ For a review, see Ref. [5].
} 
coefficients in a single, unified framework. Moore and Sohrabi [8] showed that all the 2nd-order coefficients could be expressed in terms of Kubo-like formulas provided one considers three-point retarded correlators of stress-energy tensors as well as two-point correlators. Finite-temperature retarded three-point correlators are precisely what our group had developed the technology to compute with gauge-gravity duality. We were able to calculate the complete set of 2nd-order coefficients using such Kubo formulas [AV4] (and in the process correct errors in the 3-point Kubo relations in the literature), providing a cross-check of both earlier results and of our own earlier formalism for computing three-point functions.

\section{Moving beyond leading-order supergravity}

Calculations using classical, low-energy supergravity in the 5-dimensional gravity dual correspond to the $N_{\mathrm{c}} \rightarrow \infty$ and $\lambda=N_{\mathrm{c}} g^{2} \rightarrow \infty$ limits of the 4-dimensional quantum field theory. As a result, there is an order of limits issue in studies of high energy jet-like excitations. Do formulas like $E^{1 / 3}$ and $(E L)^{1 / 4}$ for stopping distances hold at arbitrarily large energies in finite-temperature $\mathcal{N}=4$ super Yang-Mills and its cousin theories? Or do these formulas break down when $E$ exceeds, for example, $\lambda^{n} T$ or $N_{\mathrm{c}}^{m} T$ for some powers $n$ and $m$ ? We wish to explore this issue by computing the leading corrections to the stopping distance in powers of $1 / \lambda$ and $1 / N_{\mathrm{c}}$. If the relative size of those corrections remains small as $E \rightarrow \infty$, this will give some reassurance (though not proof) that the leading-order answers may be valid even at arbitrarily large energies. If the relative size of the corrections becomes large with increasing energy, that is a sign that the leading-order calculations break down at high energy. In that case, it would be interesting to learn the parametric size of the energy scale at which this happens.

There is a history of calculations of $1 / \lambda$ corrections for a variety of quantities, such as viscosity. They rely upon working out the effect on the quantity of interest of known higher-curvature corrections to the supergravity action. In [AV6], we worked out the parametric size of these corrections on our calculations of jet stopping. The result is that for some jets, the corrections are small, and the $\lambda=\infty$ results are a good approximation when $\lambda$ is large. For other jets, the expansion in higher-curvature corrections breaks down, and it is not yet clear whether $\lambda=\infty$ results are reliable.

\section{Joint publications completed in last 3 years of contract}

[AV1] E. Barnes, D. Vaman, C. Wu, and P. Arnold, Real-time finite-temperature correlators from AdS/CFT, Phys. Rev. D82 (2010) 025019 [arXiv:1004.1179].

[AV2] P. Arnold and D. Vaman, Jet quenching in hot strongly coupled gauge theories revisited: 3-point correlators with gauge-gravity duality, JHEP 10 (2010) 099 [arXiv:1008.4023].

[AV3] P. Arnold and D. Vaman, Jet quenching in hot strongly coupled gauge theories simplified, JHEP 04 (2011) 027 [arXiv:1101.2689].

[AV4] P. Arnold, D. Vaman, C. Wu, and W. Xiao, Second order hydrodynamic coefficients from 3-point stress tensor correlators via AdS/CFT, JHEP 10 (2011) 033 [arXiv:1105.4645].

[AV5] P. Arnold and D. Vaman, 4-point correlators in finite-temperature AdS/CFT: jet quenching correlations, JHEP 11 (2011) 033 [arXiv:1109.0040].

[AV6] P. Arnold, P. Szepietowski, and D. Vaman, Coupling dependence of jet quenching in hot strongly-coupled gauge theories, JHEP 11 (2011) 033 [arXiv:1109.0040].

\section{Conference Proceedings}


[AV7] P. Arnold and D. Vaman, Some new results for "jet" stopping in AdS/CFT, J. Phys. G38 (2011) 124175, [arXiv:1106.1680].

\section{P. Arnold}

\section{Jet stopping and energy loss in weakly-coupled plasmas}

Usually jet stopping in weakly-coupled gauge theories is analyzed by calculating the rate for hard bremsstrahlung and pair production. If desired, one can then incorporate these rates into some sort of Monte Carlo evolution of the shower development. Speaking of rates for individual partons to split makes little sense in the strongly coupled case, however, since individual partons are a perturbative concept. This led early researchers studying the strongly coupled problem to instead focus on the jet stopping distance, which can be defined even in the strongly coupled case through measurements of conserved densities, as depicted in fig. 2. The strong-coupling results made me realize that it would be useful and interesting to know the stopping distance in the weakly-coupled case. The LPM effect implies that this stopping distance scales as $E^{1 / 2}$ at high energy, but nobody knew the precise proportionality constant. To my surprise, numerical Monte Carlo is not necessary to work it out. Graduate student Wei Xiao, undergraduate Sean Cantrell, and I found that, for sufficiently high energies, there is a reasonably simple formula for the average stopping distance for weakly-coupled plasmas [A1]. (See publication list on p. 10 for references, such as A1, to my own recent publications.)

The other piece of work on weakly-coupled plasmas during the last three years has to do with jet stopping in finite-size plasmas. It's difficult to explain quickly, but here is the general idea. There is a general formalism for dealing with the LPM effect for bremsstrahlung or pair production in finite-sized media. However, the general formalism requires substantial numerical work to solve, and people prefer limits of the problem where simpler formulas apply. Two such limits are the "thick" and "thin" plasma limits, where relatively simple results can be derived. There had been a lot of confusion in the literature about the exact range of validity of the "thin" approximation, and why it works as well as it does. In ref. [A2], I performed a parametric analysis of the transition between the thin and thick limits which helped clarify the theory issues concerning the range of validity of the two approximations.

\section{Publications for work completed in last 3 years of contract}

(See p. 9 for publications with Vaman.)

[A1] P. Arnold, S. Cantrell, and W. Xiao, Stopping distance for high energy jets in weakly-coupled quark-gluon plasmas, Phys. Rev. D81 (2010) 045017 [arXiv:0912.3862].

[A2] P. Arnold, High-energy gluon bremsstrahlung in a finite medium: harmonic oscillator versus single scattering approximation, Phys. Rev. D80 (2009) 025004 [arXiv:0903.1081].

\section{Vaman}

\section{Introduction}

Over the last few years, one of the main directions of my research has been developing a formalism for real-time AdS/CFT computations. This work and its applications to the evolution of a highly 
energetic jet moving through the strongly coupled $\mathcal{N}=4$ superYang-Mills plasma are discussed in the separate Arnold and Vaman section.

\section{Perturbative QCD Amplitudes}

String inspired methods have been used before, leading Bern, Dixon and Kosower [11] to gain better control over the perturbative Feynman diagram calculations. The reason brute force calculations are not efficient, when applied to gauge theory, is that the individual Feynman diagrams which add up to a certain scattering process amplitude do not preserve the gauge symmetry of the theory. Quite often, the expression of a single Feynman diagram is much more involved than the actual amplitude, which is gauge-invariant. In addition to this, since the gluons carry a color index, the number of Feynman diagrams that one has to compute in the standard Feynman gauge increases tremendously with the number of external legs.

In an a recent project, together with Ed Yao (U. Michigan) I studied a very intriguing proposal of Bern and collaborators [12,13], regarding the structure of perturbative QCD amplitudes. Specifically Bern, Carrasco and Johansson (BCJ) conjectured that the color-dressed tree-level gluon amplitude can be decomposed into a sum over color factors times numerators made of kinematical invariants and appropriate products of inverse propagators, with each of the numerators obeying the same Jacobi identities as the color factors. Based on this, BCJ showed that the number of independent gluon scattering amplitudes for a process involving $n$ gluons is $(n-3)$ !. Furthermore, the graviton scattering amplitude can be computed from these BCJ numerators by squaring, in a way reminiscent of the Kawai-Lewellen-Tye relations. Our work [V1] identifies the origin of the generalized gauge transformations used by Bern et al. in obtaining the BCJ numerators via on-shell recursion. The $(n-2)$ ! color-dressed Kleiss-Kuijf gluon amplitudes can be written as a square propagator matrix times a numerator column, with the propagator matrix encoding the simple poles of the amplitude. We also pointed out that the propagator matrix has precisely $(n-3)(n-3)$ ! zero eigenvalue eigenstates. This means that one is free to shift the numerators by these null eigenvectors, in a sort of generalized gauge transformations. When making use of this freedom, to set to zero a maximal number of numerators as in [V1], various relations among the color-ordered gluon and graviton amplitudes can be obtained.

\section{AdS/CMT}

A separate application of the work done concerning real-time finite-temperature AdS/CFT was to "AdS/C(ondensed)M(atter)". This acronym refers to a new offshoot of AdS/CFT, where the duality is between a gravity theory and a strongly coupled, scale-invariant, non-relativistic theory, whose symmetries are given by the Schrodinger group. In this case, the AdS background is replaced by an appropriate deformation, which corresponds to giving a VEV to a Lorentz symmetry-breaking CFT operator. The Schrodinger theory time is identified with a light-like direction in the gravity background. The conjugate momentum to the other light-like direction is a fixed quantum number in the Schrodinger theory. In [V2], I showed that real-time correlators in the Schrodinger theory are consistently computed by taking the Fourier-transforms along a light-like direction of the gravity real-time correlators, with the latter computed according to our earlier prescription. These results are in agreement with other work when available (in this case, real-time 2-point correlators), and offer explicit 3-point real-time correlator formulae.

\section{Publications for work completed in last 3 years of contract}

(See p. 9 for publications with Arnold.) 
[V1] D. Vaman, Y.-P. Yao, Constraints and Generalized Gauge Transformations on Tree-Level Gluon and Graviton Amplitudes, JHEP 1011, 028 (2010). [arXiv:1007.3475 [hep-th]].

[V2] E. Barnes, D. Vaman, C. Wu, Holographic real-time non-relativistic correlators at zero and finite temperature, Phys. Rev. D82, 125042 (2010). [arXiv:1007.1644 [hep-th]].

[V3] E. Barnes, D. Vaman, Massive quark scattering at strong coupling from AdS/CFT, Phys. Rev. D81, 126007 (2010). [arXiv:0911.0010 [hep-th]].

[V4] E. Barnes, D. Vaman, C. Wu, All 4-dimensional static, spherically symmetric, 2-charge abelian Kaluza-Klein black holes and their CFT duals, Class. Quant. Grav. 27, 095019 (2010). [arXiv:0908.2425 [hep-th]].

[V5] D. Vaman, Y. -P. Yao, The Space-Cone Gauge, Lorentz Invariance and On-Shell Recursion for One-Loop Yang-Mills amplitudes, [arXiv:0805.2645 [hep-th]].

\section{Invited Contribution}

[V6] D. Vaman, Y. -P. Yao, "Analytic Scattering Amplitudes for QCD," Mod. Phys. Lett. A23, 847-856 (2008). [arXiv:0805.3351 [hep-th]]. 


\section{References}

[1] H. Liu, K. Rajagopal, U. A. Wiedemann, "Calculating the jet quenching parameter from AdS/CFT," Phys. Rev. Lett. 97, 182301 (2006).

[2] S. S. Gubser, D. R. Gulotta, S. S. Pufu and F. D. Rocha, "Gluon energy loss in the gauge-string duality," JHEP 0810, 052 (2008) [arXiv:0803.1470].

[3] Y. Hatta, E. Iancu and A. H. Mueller, "Jet evolution in the N=4 SYM plasma at strong coupling," JHEP 0805, 037 (2008) [arXiv:0803.2481].

[4] P. M. Chesler, K. Jensen, A. Karch and L. G. Yaffe, "Light quark energy loss in strongly-coupled N = 4 supersymmetric Yang-Mills plasma," Phys. Rev. D 79, 125015 (2009) [arXiv:0810.1985].

[5] D. T. Son and A. O. Starinets, "Viscosity, Black Holes, and Quantum Field Theory," Ann. Rev. Nucl. Part. Sci. 57, 95 (2007) [arXiv:0704.0240].

[6] R. Baier, P. Romatschke, D. T. Son, A. O. Starinets and M. A. Stephanov, "Relativistic viscous hydrodynamics, conformal invariance, and holography," JHEP 0804, 100 (2008) [arXiv:0712.2451].

[7] S. Bhattacharyya, V. Hubeny, S. Minwalla, M. Rangamani, "Nonlinear Fluid Dynamics from Gravity," JHEP 0802, 045 (2008). [arXiv:0712.2456].

[8] G. D. Moore, K. A. Sohrabi, "Kubo Formulae for Second-Order Hydrodynamic Coefficients," Phys. Rev. Lett. 106, 122302 (2011) [arXiv:1007.5333].

[9] H. Nastase, D. Vaman and P. van Nieuwenhuizen, "Consistent nonlinear K K reduction of 11d supergravity on $\operatorname{AdS}(7) \times \mathrm{S}(4)$ and self-duality in odd dimensions," Phys. Lett. B 469, 96 (1999) [arXiv:hep-th/9905075].

H. Nastase, D. Vaman and P. van Nieuwenhuizen, "Consistency of the AdS(7) x S(4) reduction and the origin of self-duality in odd dimensions," Nucl. Phys. B 581, 179 (2000) [arXiv:hepth/9911238].

[10] D. Vaman and H. L. Verlinde, "Bit strings from N = 4 gauge theory," JHEP 0311, 041 (2003) [arXiv:hep-th/0209215].

J. Pearson, M. Spradlin, D. Vaman, H. L. Verlinde and A. Volovich, "Tracing the string: BMN correspondence at finite $\mathrm{J}^{* *} 2 / \mathrm{N}$," JHEP 0305, 022 (2003) [arXiv:hep-th/0210102].

L. A. Pando Zayas and D. Vaman, "Strings in RR plane wave background at finite temperature," Phys. Rev. D 67, 106006 (2003) [arXiv:hep-th/0208066].

L. A. Pando Zayas and D. Vaman, "Hadronic density of states from string theory," Phys. Rev. Lett. 91, 111602 (2003) [arXiv:hep-th/0306107].

L. A. Pando Zayas, J. Sonnenschein and D. Vaman, "Regge trajectories revisited in the gauge / string correspondence," Nucl. Phys. B 682, 3 (2004) [arXiv:hep-th/0311190].

M. Kruczenski, L. A. P. Zayas, J. Sonnenschein and D. Vaman, "Regge trajectories for mesons in the holographic dual of large-N(c) QCD," JHEP 0506, 046 (2005) [arXiv:hep-th/0410035].

B. A. Burrington, J. T. Liu, L. A. Pando Zayas and D. Vaman, "Holographic duals of flavored N $=1$ super Yang-Mills: Beyond the probe approximation," JHEP 0502, 022 (2005) [arXiv:hepth/0406207]. 
I. Kirsch and D. Vaman, "The D3/D7 background and flavor dependence of Regge trajectories," Phys. Rev. D 72, 026007 (2005) [arXiv:hep-th/0505164].

J. T. Liu, D. Vaman and W. Y. Wen, "Bubbling 1/4 BPS solutions in type IIB and supergravity reductions on $S^{n} \times S^{n}$," Nucl. Phys. B 739, 285 (2006) [arXiv:hep-th/0412043]. J. T. Liu and D. Vaman, "Bubbling 1/2 BPS solutions of minimal six-dimensional supergravity," Phys. Lett. B 642, 411 (2006) [arXiv:hep-th/0412242].

B. Chen, S. Cremonini, A. Donos, F.L. Lin, H. Lin, J.T. Liu, D. Vaman, W.Y. Wen, "Bubbling AdS and droplet descriptions of BPS geometries in IIB supergravity," JHEP 0710, 003 (2007) [arXiv:0704.2233 [hep-th]].

[11] Z. Bern, L. J. Dixon and D. A. Kosower, "Progress in one-loop QCD computations," Ann. Rev. Nucl. Part. Sci. 46, 109 (1996) [arXiv:hep-ph/9602280].

[12] Z. Bern, J. J. M. Carrasco and H. Johansson, "New Relations for Gauge-Theory Amplitudes," Phys. Rev. D 78, 085011 (2008) [arXiv:0805.3993 [hep-ph]].

[13] Z. Bern, J. J. M. Carrasco and H. Johansson, "Perturbative Quantum Gravity from Gauge Theory," arXiv:1004.0476 [hep-th].

Z. Bern, T. Dennen, Y. t. Huang and M. Kiermaier, "Gravity as the Square of Gauge Theory," arXiv:1004.0693 [hep-th].

[14] D. T. Son, D. Teaney, "Thermal Noise and Stochastic Strings in AdS/CFT," JHEP 0907, 021 (2009). [arXiv:0901.2338 [hep-th]]. 


\section{P. Q. Hung's research activities covering the period 2009-2012.}

Two main projects that I have been involved in in the past three years are: (a) The physics of a heavy fourth generation, in particular the possibility that it could form bound states by the exchange of a fundamental Higgs doublet which is assumed to have no vacuum expectation value at tree-level (assuming tree-level scale), and, in particular, condensates which carry the electroweak quantum numbers of a Higgs doublet and which could spontaneously break the electroweak symmetry; (b) The physics of electroweak-scale right-handed neutrinos.

In carrying out these projects, my past and present collaborators are listed as follows.

For the physics of a fourth generation, my past and present collaborators include Gino Isidori (LAL, Frascati), Paul Frampton (UNC, Chapell Hill), Marc Sher (College of William and Mary, Williamsburg), Chi Xiong (Former UVA High Energy Theory postdoc), Tom Kephart and Chiu Man Ho (Vanderbilt University).

For the physics of electroweak-scale right-handed neutrinos, my past and present collaborators include Alfredo Aranda (University of Colima, Colima, Mexico), Jaime Hernandez-Sanchez (Dual C-P Institute of High Energy Physics, Puebla, Mexico).

The following UVA students are completing work on the electroweak precision constraints of the model of electroweak-scale right-handed neutrinos: Vinh Van Hoang and Ajinkya Kamat.

\section{Dynamical Electroweak Symmetry Break- ing with a heavy fourth generation}

In recent years, the possibility that a heavy fourth generation might exist has attracted a lot of attention, both from the point of view of experimental searches and from its theoretical implications concerning the origin of Electroweak Symmetry Breaking.

Although I have worked on the physics of the fourth generation as early as in 1992 [1], my interest in that subject got rekindled with a paper written with Paul Frampton [2] on long-lived quarks where we discussed various possibilities of long-lived 4th generation quarks that could be searched for at 
the Tevatron. In particular, we examined the cases when the 4th generation quarks do not mix much with the other three. This suggestion is still valid in searches for the 4th generation at the LHC. Following this work, I suggested in 1997 the possibility that a fourth generation could lead to gauge coupling unification at the two-loop level without SUSY[3]. In that paper, it was seen that gauge unification could be achieved at the "right" GUT scale to within 2 percent with a fourth generation and taking into account threshold effects at the GUT scale. These two papers were followed by a long and comprehensive review on quarks and leptons beyond the third generation written with Paul Frampton and Marc Sher [4] which has received a good number of citations in recent years. In [4], we discussed various issues related to the 4th generation, including experimental searches at the Tevatron and at the LHC.

Current LHC data [5] looking for the SM Higgs boson within the framework of the SM with 4 generations (SM4) appeared to rule out the 4th quarks for a large region of masses up to approximately $600 \mathrm{GeV}$. This result however only applies to the simplest case where there is only one Higgs boson and that the 4th quarks have SM Yukawa couplings. There is also a considerable effort in extracting bounds on the mixing of the 4th generation from data on the CKM matrix [6]. Rare B decays were used in the investigation of the effects of the fourth generation which appear at one loop levels [7].

If a fourth generation (especially a very heavy one) were to exist, what would be its implications, other than just being a heavy replica of the known families? This is the question that my former postdoc, Chi Xiong, and I asked when we tried to examine the underlying physics of a heavy fourth generation $[8,9,10]$. There were two aspects that we have investigated: a) The behaviour of the various couplings as one increases the energy scale starting from the electroweak scale; b) The behaviour of the system when the Yukawa coupling becomes large.

In [10], we studied the condition under which a condensate of the type $\left\langle\bar{t}_{L}{ }_{L} t_{R}^{\prime}\right\rangle$ gets formed. A glimpse of that condition can be seen in [8] where the Rayleigh-Ritz variational method was used, similar to the method used in [1]. In fact, bound states of the 4th generation quarks have been recently studied in [12] where an improvement of the Yukawa potential was made. The results are more precise but are not too different from those of [8].) In [10], we studied the formation of condensates by solving the SchwingerDyson (SD) equation for the self-energy of the 4th generation quarks under the ladder approximation. (This is the picture of a bound state formed by 
the exchange of a massless scalar assuming scale invariance at tree-level.) We found that the critical coupling, where the condition for condensate formation is satisfied, is $\alpha_{Y}>\alpha_{Y}^{c}=g_{Y}^{2} / 4 \pi=\pi / 2$. (This is about a factor of two smaller than the value of the quasi fixed point.) The salient point of this work is the possibility that SM4 contains the necessary ingredients to "break itself", so to say.

Phenomenologically, the Higgs spectrum has become quite interesting since one now has a scenario in which there arethree Higgs doublets, two of which are composite in addition to the original fundamental doublet. Talks on the subject: 2nd Workshop on "Beyond the 3-generation SM in the LHC era", January 14-16, 2010, Taipei, Taiwan, invited speaker; Flavour Physics: Strong Dynamics, Rare Decays and New Phenomena, October 7-9, 2010, Lake Starnberg, Germany, invited speaker; Topical conference on elementary particles, astrophysics, and cosmology, 14-19 December 2010, Miami, invited speaker; Plenary Talk, "Fourth Fermion generation and single top production 2012", 26-28 March 2012, Leinsweiler, Pfalz, Germany. Also seminars were given at Physics Department, Vanderbilt University, Nashville, Tennessee, March 5, 2010; Laboratoire d'Accelerateur Lineaire, Orsay, France, June 28, 2010; Laboratoire d'Annecy-Le-Vieux de Physique Theorique, Annecy, France, July 2, 2010; Bartol Institute, University of Delaware, Newark, Delaware, March 2011.

Finally, Chiu Man Ho and Tom Kephart and I wrote paper on some conformal models which contain SM4 beyond the TeV-scale cutoff [11].

\section{Electroweak-scale $\nu_{R}$ model}

One of the most interesting and popular explanation for the lightness of the neutrinos is the so-called seesaw mechanism in which there are two mass scales: The Dirac mass $m_{D}$ and the Majorana mass $M$. In this scenario there are, for each neutrino, two mass eigenstates: The light neutrinos (mostly left-handed active neutrinos) with masses $m_{D}^{2} / M$ and the heavy neutrinos (mostly right-handed sterile neutrinos) with masses $M$, with the former being very light when $m_{D} \ll M$. In a generic seesaw scenario, $M$ is typically of the order of some Grand Unified Theory (GUT) scale while $m_{D}$ is of the order of the charged lepton mass scales. As such it would be impossible 
to detect right-handed neutrinos and to check the viability of the seesaw mechanism directly. What if the right-handed neutrinos were not sterile i.e. not singlets under the SM group $S U(2)_{L} \times U(1)_{Y}$ ? If so then the righthanded neutrino masses would have to be of the order of the electroweak scale and, as a consequence, would be accessible at the LHC because of their masses and because they would carry the electroweak quantum numbers. This is the motivation for the construction of the model of electroweak-scale right-handed neutrinos [13].

In [13], the gauge group is still that of the SM, namely $S U(3)_{c} \times S U(2)_{L} \times$ $U(1)_{Y}$. The fermion and scalar content is now enlarged to include "mirror fermions", two Higgs triplets and a Higgs singlet. By "mirror fermions", we mean that for every left-handed doublet of SM fermions (quarks and leptons), there is a right-handed doublet of "mirror fermions" e.g. For every right-handed SM fermion $S U(2)_{L}$ singlet, there is a singlet left-handed mirror fermion e.g. $e_{L}^{M}$. Among the mirror fermions, the neutral right-handed leptons are identified as the right-handed neutrinos in this model and, as such, they are members of $S U(2)_{L}$ doublets. The mirror quarks are represented in a similar fashion.

As shown in [13], a Majorana mass term for $\nu_{R}$ is obtained by coupling $l_{R}^{M, T} \sigma_{2} l_{R}^{M}$ to a Higgs triplet $\tilde{\chi}=\left(\chi^{0}, \chi^{+}, \chi^{++}\right)$. In addition, the model contains another Higgs triplet with hypercharge $Y=0 \xi=\left(\xi^{-}, \xi^{0}, \xi^{+}\right)$such that $\left\langle\chi^{0}\right\rangle=\left\langle\xi^{0}\right\rangle=v_{M}$. This is needed in order to preserve the custodial symmetry at tree level while having $v_{M}$ of the order of the electroweak scale. The right-handed neutrino obtains a Majorana mass $\propto v_{M} \propto \Lambda_{E W}$. It is seen from [13] that the neutrino Dirac mass term is obtained through the coupling to the Higgs singlet while the charged fermions gain masses through the coupling with the SM Higgs doublet. What are the phenomenological implications of this model?

With my two UVA students, Vinh Hoang and Ajinkya Kamat, we are finishing a paper on the calculation of the parameters $\mathrm{S}, \mathrm{T}$ and $\mathrm{U}$ in the model. This is necessary to see whether or not the model is viable from the point of view of the electroweak precision constraints. Since the scalar sector in this model is more complicated (two triplets and one doublet), the contributions of this sector to $\mathrm{S}, \mathrm{T}$ and $\mathrm{U}$ are quite involved and the computations have just been completed. The experience gained in a previous (unrelated) paper [14] showed that the contribution to S from a triplet Higgs can be substantially negative, offsetting the positive contributions from chiral 
multiplets. We are finding a similar phenomenon here and the model fits quite nicely with electroweak precision data.

Phenomenologically, the rich spectrum of scalars which includes a doubly charged Higgs is a very interesting one to explore. Some preliminary investigation of the scalar sector was done in collaboration with Alfredo Aranda and Jamie Hernandez [15]. Phenomenological studies of the mirror fermion sector are underway in collaboration with Alfredo Aranda and Dilip Kumar Ghosh. This sector should have very distinctive signatures at the LHC.

\section{Conference, Summer Schools and other ac- tivities activities}

I was involved with the organization of Second Workshop on Beyond 3 Generation Standard Model-New Fermions at the Crossroads of Tevatron and LHC, January 14-16, 2010 (Taipei, Taiwan) and the Third Workshop was held in Istanbul, October 23-25,2011.

I organized and chaired a parallel session on the 4th generation at the 22nd Rencontres de Blois, Particle Physics and Cosmology: First results from the LHC, July 15-20, 2010.

Qaisar Shafi (University of Delaware) and I organized the 2011 BCVSPIN Advanced Study Institute in Particle Physics and Cosmology in Hue, Vietnam, July 25-30, 2011. The acronym of the school stands for Bangladesh, China, Vietnam, Sri Lanka, Pakistan, India, and Nepal. The 2007 and 2009 BCVSPIN schools were organized at the Graduate School of the Chinese Academy of Sciences, Beijing, Chiana. The main purpose of this summer school is to provide students from these countries the chance to be exposed to the latest advances in Particle Physics and Cosmology through lectures given by experts in the fields. It was a very successful summer school, judging from responses of students and lecturers. The website for this summer school is http://galileo.phys.virginia.edu/ pqh/2011bcvspin2.html. I have also helped in the organization of the 17th Vietnam School of Physics held in Hue on July 18-24, 2011.

I will be organizing a workshop entitled "Beyond the Standard Model: The 4th family and other exotics" in December 2012 at the International Center for Interdisciplinary Science and Education in Vietnam, Qui Nhon, 
Vietnam. This center which is founded by J. Tran Thanh Van (Rencontres de Moriond) will begin construction 16 December 2011 (where I will be present as a lecturer of the 3rd school on Physics at the LHC, http://iop.vast.ac.vn/activities/lhcs/11/).

\section{List of publications}

- Electroweak precision constraints on the electroweak-scale right-handed neutrino model, with Ajinkya Kamat, Vinh Hoang, in preparation.

- Dynamical Electroweak Symmetry Breaking with a heavy fermion in light of recent LHC results, AHEP Special Issue on Very Heavy Quarks, 2012.

- C. M. Ho, P. Q. Hung and T. W. Kephart, "Conformal Completion of the Standard Model with a Fourth Generation," JHEP 1206, 045 (2012) [arXiv:1102.3997 [hep-ph]].

- P. Q. Hung and C. Xiong, "Dynamical Electroweak Symmetry Breaking with a Heavy Fourth Generation," Nucl. Phys. B 848, 288 (2011) [arXiv:1012.4479 [hep-ph]].

- P. Q. Hung and C. Xiong, "Implication of a Quasi Fixed Point with a Heavy Fourth Generation: The emergence of a TeV-scale physical cutoff," Phys. Lett. B 694, 430 (2011) [arXiv:0911.3892 [hep-ph]].

- P. Q. Hung and C. Xiong, "Renormalization Group Fixed Point with a Fourth Generation: Higgs-induced Bound States and Condensates," Nucl. Phys. B 847, 160 (2011) [arXiv:0911.3890 [hep-ph]].

- P. H. Frampton and P. Q. Hung, "Positron Excess, Luminous-Dark Matter Unification and Family Structure," Phys. Lett. B 675, 411 (2009) [arXiv:0903.0358 [hep-ph]].

\section{References}

[1] P. Q. Hung, Phys. Rev. Lett. 69, 3143-3146 (1992); P. Q. Hung, R. McCoy, D. Singleton, Phys. Rev. D50, 2082-2108 (1994). 
[2] P. H. Frampton, P. Q. Hung, Phys. Rev. D58, 057704 (1998). [hep$\mathrm{ph} / 9711218]$.

[3] P. Q. Hung, Phys. Rev. Lett. 80, 3000 (1998) [arXiv:hep-ph/9712338].

[4] P. H. Frampton, P. Q. Hung, M. Sher, Phys. Rept. 330, 263 (2000). [hep-ph/9903387].

[5] M. M. H. Luk, "Search for Fourth Generation Quarks at CMS,"

[6] H. Lacker and A. Menzel, JHEP 1007, 006 (2010) [arXiv:1003.4532 [hep-ph]].

[7] See e.g A. J. Buras, B. Duling, T. Feldmann, T. Heidsieck, C. Promberger and S. Recksiegel, JHEP 1009, 106 (2010) [arXiv:1002.2126 [hep-ph]], and references therein.

[8] P. Q. Hung, C. Xiong, Nucl. Phys. B847, 160-178 (2011). [arXiv:0911.3890 [hep-ph]].

[9] P. Q. Hung, C. Xiong, Phys. Lett. B694, 430-434 (2011). [arXiv:0911.3892 [hep-ph]].

[10] P. Q. Hung, C. Xiong, Nucl. Phys. B848, 288-302 (2011). [arXiv:1012.4479 [hep-ph]].

[11] C. M. Ho, P. Q. Hung and T. W. Kephart, "Conformal Completion of the Standard Model with a Fourth Generation," JHEP 1206, 045 (2012) [arXiv:1102.3997 [hep-ph]].

[12] K. Ishiwata, M. B. Wise, Phys. Rev. D83, 074015 (2011). [arXiv:1103.0611 [hep-ph]].

[13] P. Q. Hung, Phys. Lett. B 649, 275 (2007) [arXiv:hep-ph/0612004]; P. Q. Hung, Contributed to 21st Les Rencontres de Physique de la Vallee d'Aoste, La Thuile, Aosta, Italy, 4-10 Mar 2007. arXiv:0706.2753 [hep-ph]. P. Q. Hung, Proceedings of the XXIemes Rencontres de Blois, Windows on the Universe, June 21-26, 2009 Blois, France.

[14] M. Adibzadeh, P. Q. Hung, Phys. Rev. D76, 085002 (2007). [arXiv:0705.1154 [hep-ph]]. 
[15] A. Aranda, J. Hernandez-Sanchez and P. Q. Hung, JHEP 0811, 092 (2008) [arXiv:0809.2791 [hep-ph]].

PA,0811,092; 


\section{Completed Research: Hank Thacker}

\section{Vacuum Topological Structure and Chiral Dynamics in Strongly Coupled Gauge Theory}

In spite of the impressive quantitative success of lattice QCD calculations, some of the basic structural properties of QCD remain poorly understood. The gauge dynamics responsible for chiral symmetry breaking, the origin of quark confinement, and the relation between them are still unresolved questions even at the qualitative level. A clearer theoretical understanding of these issues would be of great interest not only for QCD itself, but also for investigating the possibility of strong dynamics in the Higgs sector at the $\mathrm{TeV}$ scale. If no Higgs scalar is found below 600-700 GeV, unitarity arguments [1] imply a strongly interacting Higgs sector, including strongly interacting longitudinal $W$ 's and $Z$ 's. The most popular idea for the dynamics of such a strongly interacting Higgs-Goldstone sector is that it is formed from techniquark bound states in a technicolor gauge theory, with the Higgs VEV resulting from the techni- $\bar{Q} Q$ chiral condensate. Although this idea is inspired by the analogy with chiral symmetry breaking in QCD, it is now well-understood that, in order to give masses to the ordinary quarks without unacceptably large flavor changing neutral currents, the technicolor gauge theory cannot be a simple clone of QCD in terms of its renormalization group properties. Instead, it must exhibit a "walking" or "conformal window" behavior, i.e. an approximate fixed point of the beta-function at strong coupling. When combined with large anomalous dimension of the quark mass operator, this allows the extended technicolor scale to be moved high enough to avoid FCNC without making quark masses unacceptably small, as would be the case for a QCD-like technicolor theory, where the coupling constant is running rapidly at the chiral symmetry breaking scale.

Both theoretical and numerical study of the interplay between chiral symmetry breaking and renormalization group properties are needed to assess the viability of the walking technicolor idea and and to explore the range of phenomenological possibilities. If it turns out that the Higgs sector is built from a strongly coupled technicolor gauge theory, a clearer understanding of the dynamics of $\chi S B$ in gauge theories will be essential for interpreting LHC physics.

\section{Background: Topological Charge Structure in Lattice Gauge Theory}

The research completed in the past 3 years by Hank Thacker builds on developments that have emerged in studies of chiral dynamics and topological charge structure in lattice gauge theory by Thacker and collaborators. This work began in the late 1990's on the Fermilab array processor with a study of quark zero modes on the lattice and their relation to exceptional configurations [2]. In addition to providing insight into the topological origin of exceptional configurations, this work resulted in a method for handling these configurations which allowed greatly improved statistics at much lower pion mass than was previously accessible. This development led to the first clear observation of quenched chiral logs in several meson correlators [3]. These quenched chiral log effects had been predicted earlier [4], and they provide a fascinating window on the axial U(1) anomaly and topological charge structure by exhibiting the peculiar properties of the $\eta^{\prime}$ flavor singlet pseudoscalar meson in the quenched approximation. In full QCD the $\eta^{\prime}$ gets most of it's mass from topologically in- 
duced $q \bar{q}$ annihilation insertions. In the quenched approximation, this annihilation still takes place in valence-quark diagrams, but the bubble resummation of repeated mass insertions and quark loops that produces the massive $\eta^{\prime}$ propagator does not occur in the quenched theory. Instead of becoming massive, the quenched $\eta^{\prime}$ propagator contains a double massless Goldstone pole in the chiral limit. Chiral loops containing $\eta^{\prime}$ propagators exhibit striking anomalous effects in the quenched theory at small quark mass, including the appearance of Bernard-Golterman ghost states in intermediate states containing an $\eta^{\prime}$ [5].

The work with Bardeen, et al, included a study of the quenched $\eta^{\prime}$ mass insertion ("hairpin" correlator) diagram, along with a direct measurement of the topological susceptibility. The results showed that the hairpin diagram was described quite well by a pure $\eta^{\prime}$ mass insertion, with a mass consistent with the Witten-Veneziano relation. This lends support to the large- $N_{c}$ view of topological charge structure in the QCD vacuum $[8,9]$. It is noteworthy that even in full QCD, where the $\eta^{\prime}$ is massive, the hairpin diagram still has a massless pole in the chiral limit, which exactly cancels the Goldstone pole of the valence diagram. (The $\eta^{\prime}$ propagator is given by valence + hairpin, while the nonsinglet pion propagator is given by the valence diagram alone.) As discussed in the next Section, the massless pole in the hairpin diagram is a manifestation of the "hidden long-range order" in the gauge field associated with topologically nontrivial gauge configurations, as first emphasized by Luscher [10].

Following the study of the $\eta^{\prime}$ mass insertion diagram, in collaboration with I. Horvath (a UVa postdoc who is now a faculty member at U. of Kentucky) and the Kentucky lattice group, an investigation was mounted to determine the detailed structure of topological fluctuations in the QCD vacuum. The advent of exactly chiral lattice fermions satisfying Ginsparg-Wilson relations provided a new way of constructing the local topological charge density operator on the lattice, using the overlap Dirac operator. The collaboration of Thacker with the Kentucky lattice group [6] was the first study of topological charge distributions on the lattice using the overlap Dirac operator. The central result has since been confirmed by a large European lattice group [7]. What was found in both of these studies was a remarkable extended topological structure consisting of thin coherent codimension one sheets of topological charge, with sheets of opposite sign closely juxtaposed in dipole layers. Numerically, these sheets are responsible for building up the positive contact term which dominates the topological charge 2-point correlator and produces a finite, positive topological susceptibility[11]. This discovery immediately suggested a connection with the ideas about topological domain walls in the gauge theory vacuum at large- $N_{c}$ (later interpreted as D6 branes in holographic QCD) discussed by Witten $[8,9]$ as well as the closely related idea of membrane-like "Wilson bag" excitations of the 3-index Chern-Simons tensor,

$$
\mathcal{K}_{\mu \nu \lambda}=\operatorname{Tr}\left(A_{\mu} F_{\nu \lambda}-\frac{2}{3} A_{\mu} A_{\nu} A_{\lambda}\right)
$$

as suggested by Luscher [10]. According to these arguments, at large $N_{c}$, instantons are suppressed in favor of codimension one membranes which can be interpreted as domain walls between discrete quasistable "k-vacua." These discrete vacua are characterized by an effective value of the $\theta$ parameter which is displaced from zero by integer multiples of $2 \pi$. As discussed in the following Section, these can be interpreted as vacua which contain units of background Ramond-Ramond flux. The codimension one dipole sheets of topological charge observed on the lattice can be identified with domain walls between k-vacua. In this interpretation, 
they can also be described as Wilson bags, i.e. excitations defined by an integral of the 3 -index Chern-Simons tensor over the 3-dimensional surface of the bag. It is easy to show that including such a Wilson bag integral in the action inserts a boundary or domain wall, with the local value of $\theta$ jumping by $\pm 2 \pi$ across the bag surface (for an integer charged bag). (Note that when light quarks are included in the gauge theory, $\theta$ becomes identified with a $\mathrm{U}(1)$ chiral rotation, and hence the local variation of $\theta$ is identified with the flavor singlet $\eta^{\prime}$ meson field.) This topological structure in the large- $N_{c}$ vacuum of $4 \mathrm{D}$ gauge theory is greatly clarified by the analogy with 2-dimensional U(1) models, where the domain walls are just charged particles, as discussed in the next section.

The exact cancellation between the long-range part of the hairpin diagram and the Goldstone pole of the valence diagram also points to the deep connection between topological fluctuations, which produce the axial U(1) anomaly, and the gauge fluctuations which drive spontaneous breaking of $S U\left(N_{f}\right) \times S U\left(N_{f}\right)$ and cause the formation of the chiral condensate. The role of topological fluctuations as the driver of $S \chi S B$ has been suggested previously in the context of the instanton liquid model $[12,13]$. The holographic formulation of gauge theory and the recent understanding of the role of the Ramond-Ramond field $[14,15]$ provides new insight into this old question. In that analysis it was shown that 4-quark contact terms arising from RR exchange not only provide the $\eta^{\prime}$ mass insertion, but also cause the appearance of a $U\left(N_{f}\right) \times U\left(N_{f}\right)$-symmetric Nambu-Jona Lasinio (NJL) interaction. From the Monte Carlo studies it is quite clear that the topological susceptibility and $\eta^{\prime}$ mass receive their dominant contribution from the singular, codimension one topological charge dipole sheets. This suggests the interesting possibility that the $\eta^{\prime}$ mass and the chiral condensate have a common origin: the singular, sheet-like gauge configurations which are represented in the holographic framework by the RR U(1) gauge field. The NJL contact interaction arising from RR exchange can be understood as a pairing interaction resulting from the appearance of "near zero" left- and right-handed Dirac eigenmodes on opposite sides of the topological charge dipole sheets.

It should be emphasized that, in the context of $4 \mathrm{D}$ gauge theory without quarks, the Ramond-Ramond field described by the local $\theta$ parameter does not represent any additional degree of freedom beyond the Yang-Mills field. Rather it is an auxiliary field that describes the singular, sheetlike Chern-Simons excitations of the gauge field. The NJL model, in which $S \chi S B$ is driven by a short-range 4-quark interaction, is generally successful in describing chiral dynamics, but the origin of the 4-quark interaction within QCD has remained obscure. The holographic formulation and the role of the RR field in chiral dynamics suggests a topological origin of the NJL interaction.

\section{A New Paradigm for the Gauge Theory Vacuum.}

In a recent paper with UVa postdoc Chi Xiong and grad student Ajinkya Kamat, [14] the dynamics of topological charge fluctuations in the gauge vacuum and their role in chiral symmetry breaking were explored in the framework of holographic QCD and string/gauge duality. This exploration exposed the central role of the Ramond-Ramond closed-string U(1) gauge field and it's connection to the Chern-Simons tensor of the color gauge field. This is essentially a further development of the idea of Witten that the topological fluctuations of the QCD vacuum at large- $N_{c}$ should be dominated by domain walls between discrete

quasivacua. In the IIA string theory framework of the WSS model, these domain walls 
arise from D6 branes, which are magnetic sources of the Ramond-Ramond gauge field. The holographic approach provides a conceptual framework which greatly clarifies the significance of the lattice results.

One of the most promising avenues toward an analytic understanding of the D6 brane dominated vacuum in QCD is the anomaly inflow formulation, which was used in Ref. [14] to generalize the Kogut-Susskind dipole mechanism to 4-dimensional QCD. It is important to recognize that, in the presence of $\mathrm{D} 6$ branes, the $\mathrm{RR}$ 1-form potential $C_{1}$ is not globally defined (like the electromagnetic gauge potential in the presence of magnetic monopoles). The $C_{1}$ potential must instead be patched together on overlapping sections, with topologically nontrivial gauge transformations relating $C_{1}$ on different sections. Since the RR potential $C_{1}$ is only globally defined mod $2 \pi$, the term which couples $C_{1}$ to the QCD topological charge becomes well-defined only after integration by parts,

$$
\int_{D_{4}} C_{1} \wedge \operatorname{Tr} F \wedge F \rightarrow-\int_{D 4} d C_{1} \wedge \mathcal{K}_{3}
$$

where $\mathcal{K}_{3}$ is the Chern-Simons 3 -form defined by (1). Thus, the QCD $\theta$ term should be written as

$$
\int d^{4} x \partial^{\mu} \theta K_{\mu}
$$

In order for this term to be gauge invariant, the RR field $\partial^{\mu} \theta$ must transform nontrivially under a color gauge transformation to compensate for the gauge variation $\delta K_{\mu}$. This brings

a powerful set of ideas associated with gauge group cohomology and the descent equations of Yang-Mills theory [25] to bear on the problem of membrane dynamics in the QCD vacuum. Consider the variation of the CS 3 -form under a color gauge transformation, $\mathcal{K}_{3} \rightarrow \mathcal{K}_{3}+\delta \mathcal{K}_{3}$ Since the exterior derivative of the 3 -form $\mathcal{K}_{3}$ is the gauge invariant topological charge, we may assert that

$$
\delta d \mathcal{K}_{3}=d\left(\delta \mathcal{K}_{3}\right)=0
$$

Thus $\delta \mathcal{K}_{3}$ is closed, but it is not exact. It can be written as an exterior derivative of a 2-form,

$$
\delta \mathcal{K}_{3}=d \mathcal{K}_{2}
$$

but it is not exact because $\mathcal{K}_{2}$ is only defined $\bmod (2 \pi)$. The $\bmod 2 \pi$ ambiguity is similar to that of a Wess-Zumino-Witten term. The ambiguity in the solution of (5) for $\mathcal{K}_{2}$ represents the mod $2 \pi$ ambiguity associated with topologically nontrivial gauge transformations (i.e. where the gauge parameter is not single valued). From this viewpoint, the analog of quantization of the WZW coupling constant is the quantization of the step in $\theta$ across a D6 brane to be an integer multiple of $2 \pi$. The anomaly inflow idea provides a connection between the dynamics of the RR field, represented by $\partial_{\mu} \theta$, and the gauge variation of the Chern-Simons form (5). Further investigation of the anomaly inflow formulation and the use of gauge group cohomology and the descent equations to describe membrane dynamics in the QCD vacuum will be pursued in forthcoming research.

\section{Publications - 2008-2011}

1. "Marginal Boundary Perturbations and the Breakdown of the Perturbative Vacuum in Asymptotically Free Gauge Theories," H. Thacker, Talk Presented at the Sixth 
International Symposium on Quantum Field Theory and Symmetries, Lexington, KY, July, 2009.

2. "Chiral Quark Dynamics and the Ramond-Ramond U(1) Gauge Field," H. B. Thacker, PoS LATTICE2011 (2011) 259.

3. "Chiral Quark Dynamics and Topological Charge: The Role of the Ramond-Ramond U(1) Gauge Field in Holographic QCD," H. B. Thacker, C. Xiong, and A. Kamat, Phys. Rev. D84 (2011) 105011.

4. "Lattice field theory. Proceedings, 26th International Symposium, Lattice 2008, Williamsburg USA, (editor).

5. "Tachyonic crystals and the laminar instability of the perturbative vacuum in asymptotically free gauge theories," H. B. Thacker, Phys. Rev. D81 (2010) 125006.

6. "Fractionally Charged Wilson Loops as a Probe of Theta-Dependence in CP(N-1) Sigma Models: Instantons vs. Large N" Patrick Keith-Hynes and H. B. Thacker, Phys. Rev. D78:025009 (2008).

7. "Fractionally charged Wilson loops as a probe of theta-dependence in $\mathrm{CP}(\mathrm{N}-1)$ sigma models," PoS LATTICE2008 (2008) 259.

8. "Melting Instantons, Domain Walls, and large N," H. B. Thacker, PoS LATTICE2008 (2008) 260.

\section{References}

[1] B. Lee, C. Quigg, and H. Thacker, Phys. Rev. D16, 1519 (1977).

[2] W. Bardeen, A. Duncan, E. Eichten, and H. Thacker, Phys. Rev. D57, 1633 (1998).

[3] W. Bardeen, et al, Phys. Rev. D59, 014507 (1999); Phys. Rev. D62, 114505 (2000); Phys. Rev. D69, 054502 (2004).

[4] C. Bernard and M. Golterman, Phys. Rev. D46, 853(1992);S. Sharpe, Phys. Rev. D46, 3146 (1992).

[5] W. Bardeen, et al, Phys. Rev. D65, 014509 (2001).

[6] I. Horvath, S. Dong, T. Draper, F. Lee, K.F. Liu, N. Mathur, H. Thacker, and J. Zhang, Phys. Rev. D68, 114505 (2003).

[7] E. Ilgenfritz, et al., Phys. Rev. D76:034506 (2007).

[8] E. Witten, Nucl. Phys. B149, 285 (1979);ibid, B156, 269 (1979); Ann. Phys. 128, 363 (1980)

[9] E. Witten, Phys. Rev. Lett. 81: 2862 (1998).

[10] M. Luscher, Phys. Lett. B78, 465 (1978). 
[11] I. Horvath, et al, Phys. Lett. B612, 21 (2005); ibid, B617, 49 (2005).

[12] R. Carlitz and D. Creamer, Ann. Phys. 118, 429 (1979).

[13] D. Diakonov and V. Petrov, Phys. Lett. B147, 351 (1984).

[14] H. Thacker, C. Xiong, and A. Kamat, Phys. Rev. D (2011) (to be published), arXiv:1104:3063 [hep-th].

[15] H. Thacker, Phys. Rev. D81, 125006 (2010).

[16] S. Ahmad, J. Lenaghan, and H. Thacker, Phys. Rev. D72: 114511 (2005).

[17] Y. Lian and H. Thacker, Phys. Rev. D75: 065031 (2007).

[18] P. Keith-Hynes and H. Thacker, Phys. Rev. D78:025009 (2008).

[19] S. Coleman, Ann. Phys. 101, 239 (1976).

[20] M. Green, J. Harvey, and G. Moore, Class. Quant. Grav. 14, 47 (1997).

[21] T. Sakai and S. Sugimoto, Prog. Theor. Phys. 113, 843 (2005).

[22] P. Di Vecchia and G. Veneziano, Nucl. Phys. B171: 253 (1980);

[23] C. Rosenzweig, J. Schechter, C. Trahern, Phys. Rev. D21:3388 (1980).

[24] G. Veneziano, Nucl. Phys. B159, 213 (1979).

[25] L. Faddeev, Phys. Lett. B145, 81 (1984).

[26] W. Bardeen, C. Leung, and S. Love, Nucl. Phys. B323:493 (1989). 


\section{Introduction}

\section{University of Virginia Task B Grant Closeout (April 15, 2009- May 1, 2012)}

Task B activities and Task B supported personnel are almost 100\% devoted to the CMS experiment with only residual $\mathrm{KTeV}$ analyses still on-going in the past three years. The Task B group has major responsibilities in the management, maintenance, operation and upgrade of the CMS electromagnetic detector (ECAL) in both the near and long term. We have also taken on projects for CMS HCAL.

As well as maintenance and upgrade of the ECAL detector, Task B personnel have been very active in various physics analyses including Supersymmetry, Higgs, Top, and QCD analyses.

\section{Task B 2009-12 Personnel}

The UVa personnel listed below in Table I were supported and were active on the CMS experiment during 2009-2012

Table I

UVa CMS Personnel

Faculty

B. Cox (Prof.)

R. Hirosky (Assoc. Prof)

C. Neu (Assistant Prof)

S. Conetti (Res. Prof.)
Research Associates

and Scientists

A. Ledovskoy

M. Arenton

S. Boutle
Grad Students

B. Francis

C. Lin

J. Wood

R. Yohay

J. Goodell
*Cryo. Support

A. Tobias
**Electrical Support

S. Goadhouse (EE)

L. Yao (Electrical Tech)
** Mechanical Shop

4 machinists

- Technical support is provided by project funds.

CMS graduate students, Michael Balazs (PhD in 2011), Rachel Yohay (PhD in 2012), Chuanzhe Lin, Brian Francis, John Wood, and Joey Goodell, have been very involved with CMS and have all been in residence at CERN for various periods. Rachel Yohay has spent over four years at CERN on CMS first as an undergraduate at Caltech for a summer and then as 
graduate student at UVa preparing software and hardware for the ECAL LED stabilization and calibration system. She has received special notice by CMS for her work, being one of 5 CMS students out of 831 to receive a CMS Achievement award. She also received a Advancing Science in America Foundation Scholar Award this past summer. Michael Balazs has spent periods at CERN and was the main player in developing the 3.8 Tesla (VPT) test bed at UVa until John Wood took over from him in the summer of 2010. Brian Francis modified the VPT test bed to allow for an simultaneous evaluations of multiple VPT system. Chuanzhe Lin has been active in development of prototype controller for future $\mu \mathrm{TCA}$ readout electronics for ECAL/HCAL. He has been stationed at CERN for a period this past year working on applying corrections to the signals from the ECAL endcaps. Brian Francis has been responsible for the LED system interface to the conditions database for the DAQ at CERN this past summer and has recently been the lead student on modification of the CMS FASTSIM to accommodate radiation damage effects such as transparency loss in the $\mathrm{PbWO}_{4}$ crystals, enabling realistic aging and resolution studies. Joey Goodell has been one of the main students active in doing pileup studies for the ECAL in anticipation of the higher luminosity operation of the LHC. He, along with Francis, has also been a participant in the GaAs chip development.

\section{Task B Leadership and Accomplishments}

The UVa Task B group members have served and continue to serve in many leadership roles on CMS, D0, and other HEP activities. A list of these roles is given below:

\section{B. Cox}

- is the UVa CMS team leader.

- is the Level II manager 2009-present for the US CMS Electromagnetic Detector effort (responsible for determining and administering the US ECAL budget: ( $\$ 1,185 \mathrm{~K}$ requested for FY12 for the US CMS ECAL institutions: Caltech, Princeton, Minnesota, Cornell, Florida State, Carnegie-Mellon, Kansas State, Notre Dame, M.I.T. and Virginia). The primary responsibility of a level II manager is developing an annual budget for the US ECAL groups. Among the other various duties of a Level II manager are approving the ECAL Statements of Work, submitting material for the US CMS Op. Program quarterly reports, responding to various requests both from US people working on ECAL and from the M\&O manager), preparation for the Joint Oversight Group (JOG) review, conducting mid-term budget reviews, early spring, fiscal year budget reviews, summer and long term planning for operations. 
- is the co-leader of the Joint ECAL-HCAL Forward Calorimetry Upgrade Task Force responsible for making and implementing a joint design for the upgrades of the ECALHCAL forward calorimetry. This task force is CMS-wide and includes a significant fraction of ECAL and HCAL. Its charge is to develop a design motivated by physics that is radiation hard enough to survive $3000 \mathrm{fb}^{-1}$ of integrated luminosity.

- $\quad$ is a member of the ECAL Steering/Finance committee, ECAL Institution Board and CMS collaboration board.

- $\quad$ is a member of the CMS ECAL Laser Task Force formed to decide on new calibration lasers for ECAL.

- $\quad$ is a member of the four member Higgs $\rightarrow \gamma \gamma$ and the Fermiophobic Higgs $\rightarrow \gamma \gamma$ Analysis Review Committees (ARCs) responsible for oversight of these analyses with the additional responsibility of allowing them to go forward. There were

- was the editor for the di-photon supersymmetry analysis notes AN-10-168, AN-10-271 and Physics Analysis Summary (PAS SUS-10-002): (Search for Supersymmetry in pp Collisions at $7 \mathrm{TeV}$ in Events with Two Photons and Missing Transverse Energy, Phys. Rev. Lett. 106 (2011) 211802).

- was the chair of the APS Publications Oversight Committee.

- was a member of the APS Publication Task Force on future of publications.

- is the Membership Committee coordinator of the Southeastern Section of the APS

- was a vice chair, chair-elect and chair of the Southeastern Section of APS (Past Chair).

- $\quad$ is an observer to the APS Council.

- $\quad$ is the present secretary of the Southeastern Section of the APS.

- $\quad$ is a member of the International Organizing Committee, Heavy Quark and Lepton Conference:

- at Frascati (October 11-15, 2010).

- at Prague, Charles University (June, 2012).

- At Mainz University (September, 2014)

\section{R. Hirosky}

- is the deputy UVa CMS team leader.

- $\quad$ is the head of the D0 Level 2 trigger group for Run 2.

- $\quad$ is a member of D0 Speakers Bureau. 
- is the Chair of D0 jet energy scale certification board.

- is a member of the D0 Collaboration Institutional Board.

- is a member of the D0 Editorial Boards (QCD, EW/Higgs Physics).

- is a member of the CMS Higgs $\rightarrow \mathrm{ZZ} \rightarrow 2$ lepton 2jets ARC.

C. Neu

- is the $2^{\text {nd }}$ Deputy UVa CMS Team Leader.

- is the CMS Top physics analysis group (PAG) cross-sections subgroup co-convener: This is a subgroup of the Top PAG that handles all ttbar cross section measurements in all channels and related searches.

- is the co-convener of the ttH subgroup of the Higgs Cross Sections working group: This is a working group designed to ensure consistent treatment of the Higgs cross sections is used in CMS and ATLAS. He is the CMS representative for the ttH subgroup, along with representatives from ATLAS and theory

- is the ARC chair for EWK-10-003 ("Measurement of the lepton charge asymmetry in inclusive $\mathrm{W}$ production in pp collisions at $\sqrt{s}_{\mathrm{s}}=7 \mathrm{TeV}$ ", J. High Energy Phys. 04 (2011) 050).

- is the ARC chair for EWK-11-005 ("Measurement of the Muon Charge Asymmetry in Inclusive W Production").

- is the ARC chair for EWK-11-013 ("Measurement of W+charm").

\section{A. Ledovskoy}

- is the co-leader of the ECAL Endcap Detector Performance effort.

- is the leader of the Forward Calorimetry Simulation group which is charged with simulating different configuration of the forward calorimetry for the Hi-LHC era.

- is a member of the four member Higgs $\rightarrow \gamma \gamma$ and Fermiophobic Analysis Review Committees (ARCs).

- is co-leader of the ECAL Working Group 3 which is formed to study fundamental crystal and monitoring issues, e.g. the measurement of light loss and correlate it with known crystal properties, vacuum phototriode (VPT) properties, damage mechanisms.

\section{S. Boutle}

- serves as general ECAL detector expert on-call (DOC). The DOC provides primary response for all ECAL-related issues during both in-store and out-of-store periods. This is a mission 
critical position requiring 24/7 responsiveness and essentially constant engagement.

- is one of only three ECAL DAQ experts for the entire CMC experiment.

- Is the developer of expert-level interface to database responsible for recording necessary user defined conditions for ECAL operations fulfills a role as data base editor.

Also important to mention is the accomplishments of the UVa graduates students thus far.

\section{R. Yohay and M. Balazs}

- R. Yohay was selected as one of five graduate students out of $831 \mathrm{grad}$ students on CMS to receive a CMS Achievement Award in 2011.

- R. Yohay recently received the Advancing Science in America Foundation Scholar Award.

- M. Balazs was the first UVa graduate student to receive a PhD from CMS and one of the first in CMS to take a degree in a SUSY analysis.

\section{Task B Projects}

The University of Virginia group continued to be one of the most important institutions in the US ECAL community with many responsibilities. During the preceding three-year grant, UVa maintained three to four people at CERN. We need to keep this level of participation in the upcoming three years. As well as the projects mentioned below, we are required by CMS to provide 36 man month $(\mathrm{mm})$ of ESP (service). In addition, in the 2011 calendar year we provided $4.4 \mathrm{~mm}$ of ECAL shifts and $2.3 \mathrm{~mm}$ of Central shifts. We expect to be asked to provide the same amount in grant year 2012-2013 and going forward. The time line for each aspect of the activities are discussed in the project sections. The projects accomplished in the preceding three years and continuing into the next three year grant period both at CERN and at UVa are given below. The UVa personnel involved with a given project are also listed.

\section{a) Installing and commissioning of the ECAL endcaps (Ledovskoy plus UVa Students):}

During the construction, installation, and commissioning stages of the endcaps, Alexander Ledovskoy was crucial to their success. 14,468 $\mathrm{PbWO}_{4}$ crystals were mounted on the four endcap "dees" and instrumented with their electronics. Following the preparations of the dees, Ledovskoy was instrumental in the installation of the endcaps. He continues to this time to be involved with the continuing commissioning of 
the endcaps. In particular, he has developed the technique for extracting time resolutions of better than $100 \mathrm{ps}$ for the $\mathrm{PbWO}_{4}$ crystal detector elements thereby allowing reduction of backgrounds among other improvements in the analyses. A picture demonstrating the complexity of the endcaps is shown below:

Fig. 1: Work proceeding during the construction of the ECAL endcap. The three UVa students are installing fiber optics to carry calibration light to each of the $14,468 \mathrm{PbWO}_{4}$ crystals
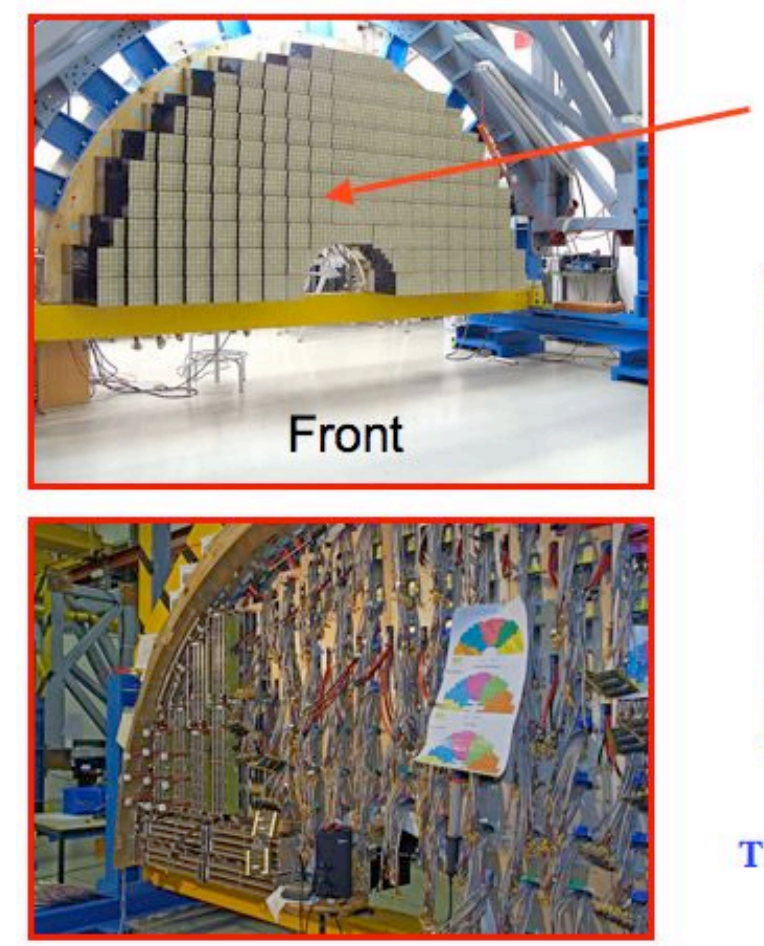

\section{One quarter (dee) of the \\ ECAL Endcaps}

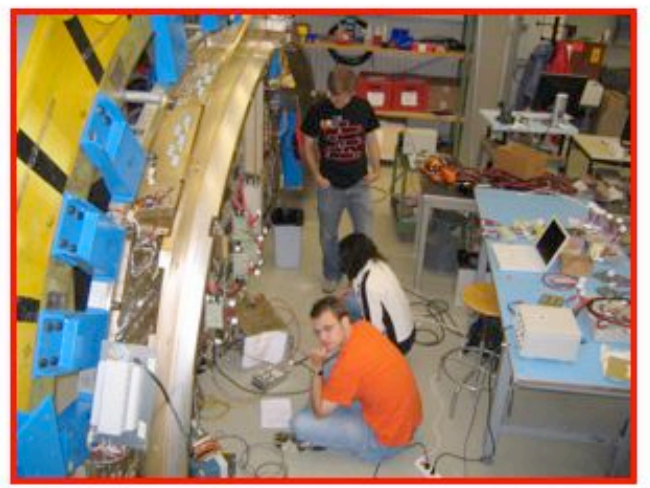

Three UVa students work on the endcaps from top: Balazs, Yohay, Andelin

\section{Rear}

Time line: The maintenance and upgrade phases of the endcap effort is on-going and indefinite. We will continue to be responsible for the maintenance of this system for the lifetime of CMS as far as currently understood.

b) The construction, installation, and operation and analysis of the UVa blueorange LED calibration and stabilization system for the ECAL endcaps 


\section{(Ledovskoy, Arenton, Yohay, Hirosky, and Lin):}

Installation of the blue-orange LED flasher system to calibrate and stabilize the 14,468 vacuum phototriodes (VPTs) of the endcap supercrystals was a delicate procedure. It required not only mounting and instrumenting the LED flasher system but also routing of fiber optics to each of the $\mathrm{PbWO}_{4}$ crystals. This system composed of 546 blue and orange LEDs functions as a device to provide fast light pulses to the crystals to stabilize the gains of the VPTs when the beam is off. The LED system and associated controls were designed and built at UVa. In addition, the LED system has served as a backup blue light $(440 \mathrm{~nm})$ calibration system for the blue laser system when needed. The LED system also provides orange light $(617 \mathrm{~nm})$ that is intended to allow separation of the effects of radiation damage on transparency loss in the $\mathrm{PbWO}_{4}$ crystals from the loss of gain in the VPTs due to the amount of charge passing through them. The red laser that is part of the laser calibration system cannot be detected in the endcaps due to the quantum efficiency of the VPTs for long wavelengths so the orange LEDs are the only option for the separation.

\section{c) Software development for setup and control of the UVa LED calibration and stabilization system. (Yohay):}

The connection of the LED system controls and the insertion of the LED data into the event data stream required development of a significant amount of hardware and software. The LED calibration data had to be inserted into the few-microsecond abort gaps, but avoid a significant part of the abort gap in which some experimenters were looking for long lived particles that decayed long after the primary interactions.

\section{d) Development of a reference design for $\mu$ TCA module management controller (MMC) (Hirosky, Goadhouse, and Lin):}

We have completed the hardware design for a uTCA-card MMC providing extended functionality including: ethernet access from backplane to on board microcontroller, local memory for storage of configuration or monitoring data, and a managed 6 (or 10)-port Gb switch to connect on board devices to the backplane or front panel. The MMC is designed to reside on a mezzanine card using a PCI Express $4 \mathrm{x}$ 
connector to mount on a uTCA (AMC) module and is compatible with the miniCTR cards in development for the HCAL readout in the Phase 1 upgrade. The design is generic and may be applied in future module development. The production of hardware prototypes and control software is presently on hold to work on more critical path tests for commissioning of ngCCM (next item). The project is ready to go to prototypes on demand. Fermilab has provided $\$ 60 \mathrm{~K}$ of funding for the $\mu$ TCA project.

\section{e) Commissioning of a Clock-Control-Module (ngCCM) for the new HCAL readout electronics (Hirosky, Goadhouse, student):}

This module is a replacement for the existing CCM cards in the HCAL detector readout. The CCM controls readout and calibration modules in the barrel and endcap detectors. It forms an interface between CMS DAQ/CONTROL and the HCAL front end, to receive and distribute the $40 \mathrm{MHz}$ LHC clock, perform slow monitoring of FE boards, and to provide a communication/download path to FE cards. The upgraded ngCCM offers faster communication via high-speed GBT links, cleaner clocks, and added redundancy to insure high data taking efficiency. For the initial prototypes of the cards the radiation-tolerant GPT link ASICs are not available. UVa will initially commission the optical links using an on board Xilinx FPGA to simulate the GBT ASIC, develop the mechanism for remote programming of the boards via the optical links, validate $8 \mathrm{~b} 10 \mathrm{~b}$ firmware for the GBT design, setup tests to measure bit-error rates and clock jitter, and participate in programming of control and monitoring software. We will also participate in in-situ commissioning at the HCAL test beam and at CMS. This work is in collaboration with Maryland, Minnesota, and Alabama.

\section{f) Operating and upgrading the 3.8T superconducting solenoid test bed at UVa (Wood, Francis, Cox):}

We have installed and operated a large bore, 3.8T superconducting solenoid test bed at UVa for purposes of testing various components of CMS in high fields. Using this test bed, Wood and Francis are performing a number of long term tests (of order many months) on the VPT's to determine the performance of the VPTs for long periods of time at high light intensity in the $3.8 \mathrm{~T}$ field of the CMS experiment. They are also 
determining the stabilization of the gain of the VPT's in high magnetic fields at different luminosities as a function of the intensity of the stabilizing light (soak light) from the blue-orange LED flasher system.

In the past grant period hundreds of VPTs were measured to determine their response to the orientation of their axes to the direction of the solenoidal magnetic field. We anticipate receiving other devices from CMS to test for sensitivity to magnetic field since this test bed with its $40 \mathrm{~cm}$ diameter aperture and its ability to reach $3.8 \mathrm{~T}$ is the only such test bed in CMS. Rutherford Appleton Laboratory (RAL) provides approximately $\mathbf{\$ 3 0 K}$ per year to partially support this test bed. Fermilab has provided \$54.5K in 2011 for continuing development of this test bed.

\section{g) Upgrading and maintaining the UVa Tier III computing facilities (Hirosky, Balazs, and Arenton):}

We have a large dedicated computing facility with 250 modern CPU cores and approximately $100 \mathrm{~TB}$ of disk storage for CMS and D0 computing. We are attached to Lambda Rail Internet 2 with a $10 \mathrm{~Gb}$ uplink capability. We also have links to considerable university based computing. Hirosky assisted by Balazs and Arenton developed over the last few years the CMS computing facility that is now a member of the Open Science Grid. We received \$46.1K in equipment funds from State of Virginia, the bulk of which has gone to increasing our computing capabilities. In addition we received $\$ 60 \mathrm{~K}$ in Recovery Act funding. These funds allowed us to purchase UPS capacity adequate to provide clean power to the analysis farm we have assembled, to increase redundancies for critical hardware, and to further expand our computing capacity which is coming under increasing stress to provide for all our physics analyses (see below) as luminosity of CMS increases.

h) R\&D for development of a GaAs radiation hard photo-detector (Hirosky, Cox, Neu, Arenton, Francis, Goodell, Yao, Goadhouse).

The objective of this project is the development of radiation hard solid-state photodetectors for ECAL and HCAL. These detectors are based on pixilated GaAs and/or GaInP chips operating in the Geiger mode and have neutron radiation hardness 
orders of magnitude higher than similar silicon devices. In addition to their radiation hardness, they have the potential to be tuned to be sensitive to different wavelength regions, thereby giving an opportunity to discriminate between prompt, short wavelength Cerenkov radiation and scintillation radiation. This in turn would provide an opportunity to determine and correct for differences in the EM and hadronic portions of given showers to produce a compensating EM-Hadronic calorimeter even with very different structures for the EM and hadronic parts. This would greatly enhance the jet energy resolution of the ECAL-HCAL combination in CMS.

CMS has approved this R\&D and US CMS has provided \$121.6K of funding for this effort to the UVa. HEP group. In addition, $\mathbf{\$ 1 0 3 K}$ for the next few years will be provided out of the chip designer company LightSpin Inc.'s SBIR's for radiation testing in hadron beam of the GaAs (and GaInP) photodetectors. Finally, a generic R\&D funding proposal on the order of $\$ \mathbf{9 6 5 K}$ to UVa to pursue not only GaAs but much more rad hard GaInP photodetectors for CMS has been submitted to DOE.

\section{i) Development of an intense blue laser diode light source that might be used eventually as a backup for the ECAL barrel laser system (Arenton):}

Our objective is to develop a laser diode driven intense blue light source to operate as a backup (or even as a replacement) for the calibration blue lasers presently in operation in CMS ECAL. The LED system in the ECAL endcaps has proved that LEDs are competitive with, much less expensive, easier to use, and more robust than lasers. Hence we are trying to develop a similar system for the ECAL barrel.

The difficulty is that, due to the small optical fiber ( $400 \mu \mathrm{m}$ diameter) feeding the barrel $\mathrm{PbWO}_{4}$ elements, we need a total power of $0.2 \mathrm{~mJ}$ from our laser diode setup. This is because that the single fiber's light is split and funneled into $900 \mathrm{PbWO}_{4}$ through two sequential diffusing spheres. To reach the requisite power needed to deliver a light pulse adequate to calibrate the crystals, we need a system that adds the light from a large number of laser diodes.

The solution presently being pursued is to use a parabolic mirror and a lens system to focus a collection of laser diodes into a $400 \mu \mathrm{m}$ fiber. A prototype has been developed. Fermilab has provided $\mathbf{\$ 3 5 . 3 K}$ for this project. 


\section{j) Simulation of pileup and radiation damage in CMS ECAL detector} (Ledovskoy, Francis, Cox, Neu, Goodell):

We are attempting at UVa to introduce real measurements of light loss due to hadronic damage in the endcap $\mathrm{PbWO}_{4}$ crystals to determine when the endcaps cease to be useful and when the radiation damage becomes comparable with the pileup degradation of the "beyond the SM" signals. An important aspect of this is the determination of when additional luminosity no longer helps the physics studies. Preliminary studies show that after $2000 \mathrm{fb}^{-1}$ of integrated luminosity, the ability of the CMS calorimeter to measure properly missing transverse energy and the direction of forward hadronic jets is compromised significantly for the present detector. The pileup studies were performed by Neu assisted by Goodell, and the radiation damage studies by Ledovskoy assisted by Francis and Cox.

\section{Physics Activities}

Our physics interests are almost totally devoted to collider physics (LHC and some D0). The three faculty members, Cox, Hirosky and Neu lead different physics projects covering wide area of physics at the LHC. CY2010 saw the accumulation of a data sample corresponding to approximately $36 \mathrm{pb}^{-1}$; in CY 2011 the data sample swelled to more than $5 \mathrm{fb}^{-1}$. The UVa CMS analysis efforts are focused on this large 2011 data sample in a suite of crucial measurements and searches.

\section{IVa. A Search for the general gauge mediated supersymmetry (GGM SUSY) beyond the SM (Balazs, Yohay, Francis, Cox, Arenton and Ledovskoy):}

The SUSY GGM mechanism is anticipated to produce combinations of gluinos or squarks from gluon-gluon interactions in 7 and $8 \mathrm{TeV}$ proton-proton collisions. Some of these SUSY particles will then decay to neutralinos plus jets followed by the decay of each of the neutralinos to a gravitino plus photon. This decay chain will result in events with two photons, large missing transverse energy (MET), and jets. We have analyzed 5 $\mathrm{fb}^{-1}$ from the 2011 data and produced a paper that is in the CWR process. In 2012 we analyzed and additional $4 \mathrm{fb}^{-1}$ in the continuation of this search. 
The early 2010 data totaling $35.5 \mathrm{pb}^{-1}$ was the basis for Balazs's, PhD thesis, which was one of the very first SUSY theses. The $5 \mathrm{fb}^{-1}$ accumulated thus far in 2011 was used for Yohay's PhD thesis. Francis's thesis should use the data from entire first run of CMS that will end in 2013.

We were involved in preparatory studies for several years for this mode,

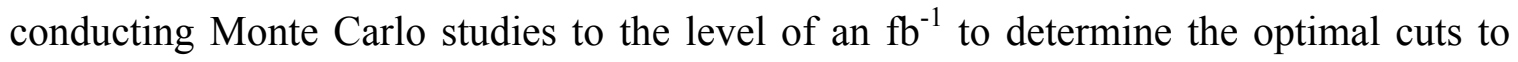
apply to preserve signal and eliminate background. We followed up this work with an analysis of the first $36 \mathrm{pb}^{-1}$ of 2010 data as mentioned. This work has resulted in the several conference presentation and CMS notes:

"Understanding Missing Transverse Energy in Di-Photon Events For SUSY Searches" CMS Physics Analysis Note 94;

"Understanding Missing Transverse Energy in Di-Photon Events in $7 \mathrm{TeV}$ Interactions" CMS Physics Analysis Note 168;

“A Search for Strong Production of New Physics in Proton-Proton Collisions at s $\mathrm{s}^{1 / 2}=7$ TeV using Events with Two Photons and Missing Transverse Energy" CMS Physics Analysis Note 271.

"A Search for Supersymmetry in Proton-Proton Collisions at $\mathrm{s}^{1 / 2}=7 \mathrm{TeV}$ using Events with Two Photons and Large Missing Transverse Energy" CMS Physics Analysis Summary.

Cox was the editor of the last three of these documents. The last two documents were steps to a Phys. Rev. Letter article "Search for Supersymmetry in pp Collisions at $=7 \mathrm{TeV}$ in Events with Two Photons and Missing Transverse Energy, Phys. Rev. Lett. 106 (2011) 211802) based on the first $35.5 \mathrm{pb}^{-1}$ of data. This past year we have taken $5 \mathrm{fb}^{-1}$ to date and $1.14 \mathrm{fb}^{-1}$ has been reported on at the August Lepton-Photon Conference. The results of the $1.14 \mathrm{fb}^{-1}$ data are shown in Fig. $2 \mathrm{a}$ and $2 \mathrm{~b}$ below.

Fig 2 A: Comparison of the data driven background estimates for missing $\mathrm{E}_{\mathrm{T}}$ for diphoton events with the di-photon spectrum 


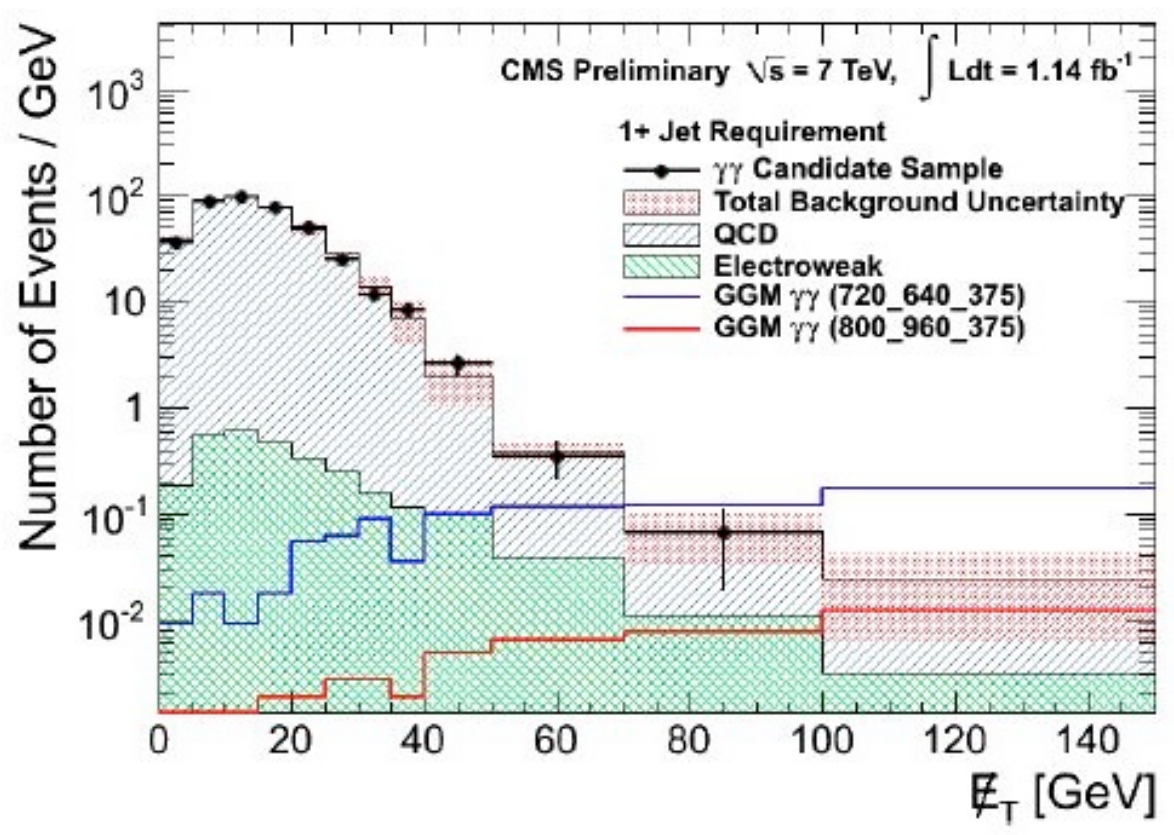

Fig. 2B: Lepton-Photon Conference exclusion plot for squark vs. gluino masses (375 $\mathrm{GeV} / \mathrm{c}^{2}$ neutralino) for GGM SUSY production determined di-photons plus large Missing $\mathrm{E}_{\mathrm{T}}\left(1.14 \mathrm{fb}^{-1}\right)$.

\section{Double photon search}

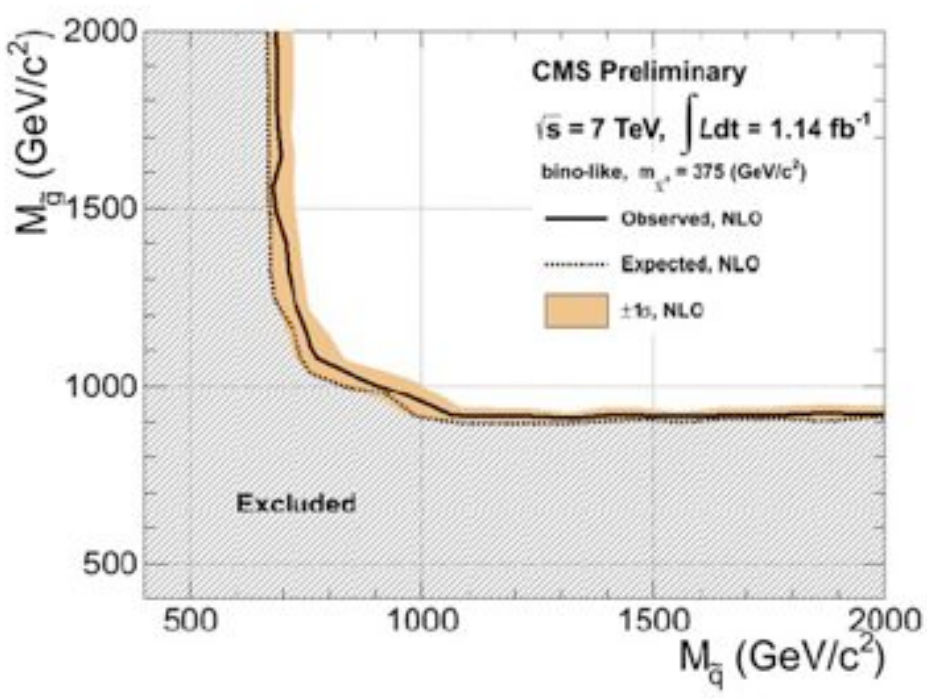

Finally, one of us (Ledovskoy) has also examined di-photon events where the photons are non-prompt due to finite lifetime of the neutralinos. This study is described in EXO- 
11-067. The Physics Analysis Summary for this study has been recently approved.

\section{IVb. Measurement of the Production Cross Section for Top-Quark Pairs} (Neu and Boutle):

Upon joining the UVa CMS effort in 2008, Neu brought considerable knowledge on top-quark production and $\mathrm{W}+\mathrm{b}$-jet signatures in general. Neu and Boutle made significant contributions to the top-quark physics program in 2009-10, including contributions to the offline trigger DQM, exercises in the muon+jets event selection, studies of optimized signal extraction techniques, studies of $\mathrm{W}+$ jets background enriched data samples and determination of an optimal QCD background model. We have been main developers of the simultaneous $\mathrm{QCD}, \mathrm{W}$, and top measurement technique (SQWaT) which was first deployed in a measurement of the top-quark pair production cross section in the $36 \mathrm{pb}^{-1}$ sample collected in 2010. Our contributions to that measurement are documented in CMS AN-10-326, "Simultaneous QCD, W, and Top Cross Section Measurement". The CMS measurement of this cross section was published in Eur. Phys. J. C71 (2011) 1721 [arXiv:arXiv:1106.0902 [hep-ex]]. The SQWaT result was not the official CMS reference result for this channel in this paper; however, our result was of comparable sensitivity with respect to the reference, and our personnel played an important role in its preparation and review. Given this role, we are included as primary authors of this paper.

In the $5 \mathrm{fb}^{-1} 2011$ data sample, the first focus of the UVa personnel in top-quark physics is to measure the differential production cross section for top-quark pairs. This large statistics sample offers experimenters the opportunity to study top-quark pair production in unprecedented kinematic regimes. Given the unique nature of the top quark within the standard model, it is imperative to study all of its properties closely, including the kinematics of its main production mechanism. Consider a heavy new state that couples to top quarks, a provocative possibility given the large value of the top quark's mass. Production of this new state could be identified through s-channel type decays to top quarks by looking for evidence of a resonance in the $\mathrm{M}_{\mathrm{tt}}$ distribution. However, if produced through t- or u-channel type diagrams, the presence of this new state may be identifiable only through subtle effects on the kinematic dependence of top- 
quark pair production. Therefore these precision measurements of top-quark pair production could in fact provide a window to evidence for new physics, a kind of search within a measurement.

The measurement of the top-quark pair differential production cross section in the 2011 sample is currently underway, in close collaboration with colleagues from Notre Dame and FNAL. We expect this result to be ready for 2012 winter conferences, and a paper will follow immediately. Neu and his personnel plan to use these experiences as a stepping off point for searches in the lepton+jets channel, pursuing signatures of Higgs, SUSY and other exotica in the coming years.

\section{IVc. Search for Standard Model Higgs Production in the Channel $\mathbf{H} \rightarrow \mathrm{WW} \rightarrow$ lvjj (Neu, Boutle, Goodell, Wood):}

In the 2010 data sample, the focus of the CMS physics program was the rediscovery of the standard model - demonstrating to the high energy physics community that the CMS experiment was functioning and could identify expected though complicated signatures, such as that of top-quark production. For the larger 2011 data sample, the focus shifted instead to searches for new physics. One of the main goals of CMS was a thorough scan of the Higgs mass parameter space; from Fig 2 one can see that with a data sample corresponding to $5 \mathrm{fb}^{-1}$ collected at $8 \mathrm{TeV}$, the CMS experiment achieves SM-level sensitivity to Higgs production throughout the range $114<\mathrm{M}_{\mathrm{H}}<600$ $\mathrm{GeV} / \mathrm{c}^{2}$. Of course the collision energy for the data sample collected in 2011 was $7 \mathrm{TeV}$, however the projections for $7 \mathrm{TeV}$ is very similar to the projections for $8 \mathrm{TeV}$. A few things should be noted: no single channel possesses SM-level sensitivity throughout the entire range of $\mathrm{M}_{\mathrm{H}}$ values considered here, necessitating a combination of all available channels; at high mass values, the Higgs sensitivity is driven by $\mathrm{H} \rightarrow \mathrm{ZZ}$ mode searches; and finally, missing from this suite of Higgs analyses is a search in the channel $\mathrm{H} \rightarrow \mathrm{WW}$ $\rightarrow$ lvjj, the semileptonic WW mode which, though challenging in terms of backgrounds, benefits from enhanced rate of signal events when compared to modes focusing on one or more leptonic $\mathrm{Z}$ decays.

Fig 3. Projected sensitivity to standard model Higgs production as a function of Higgs 
mass, assuming a data sample corresponding to $5 \mathrm{fb}^{-1}$ at $\operatorname{root}(\mathrm{s})=8 \mathrm{TeV}$. Though unavailable at this time, projections for $7 \mathrm{TeV}$ paint a similar picture.
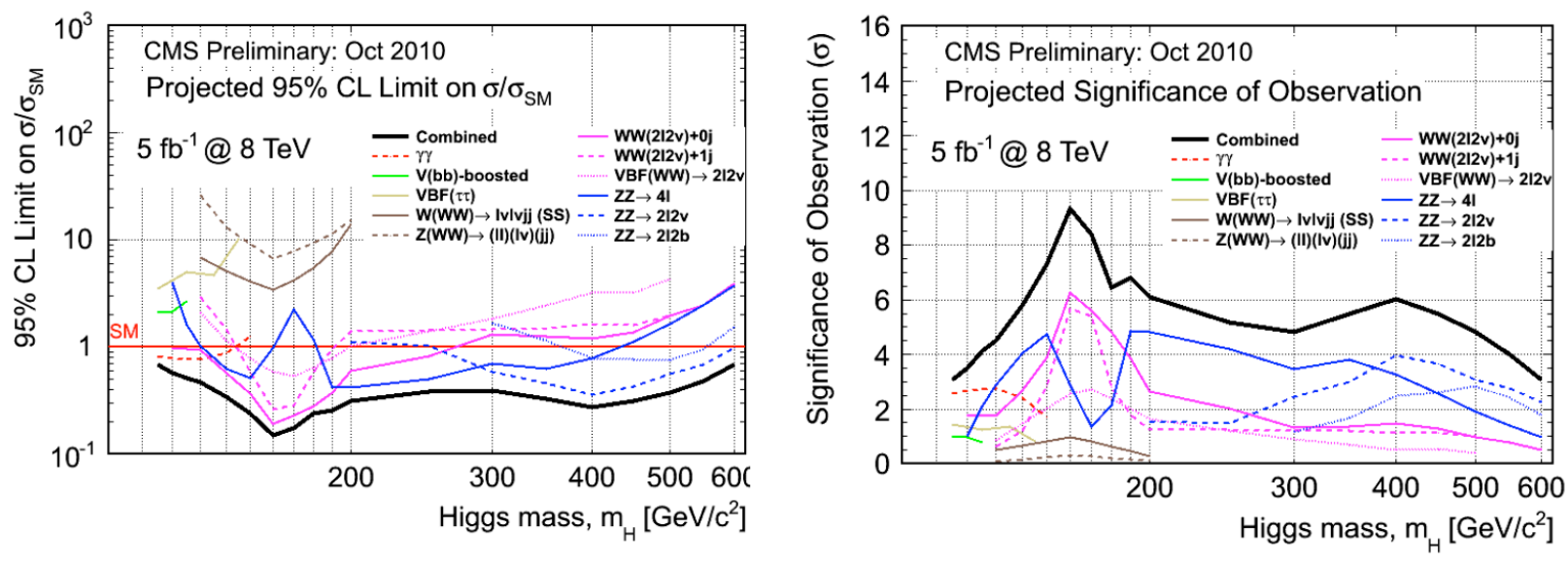

Given their experience in the Top PAG in 2010-11, Neu and his students were well positioned to pursue the Higgs search in this channel. The opportunity was identified and the analysis was initiated in April, 2011. Other groups with related expertise sought to get involved as well. A working group focusing on the $\mathrm{H} \rightarrow \mathrm{WW} \rightarrow$ lvjj was formed in May, 2011. This working group consists of representatives from three analysis groups: Milano, FNAL and UVa-Notre Dame. From the beginning, postdoc Sarah Boutle was the driving force behind determining a baseline selection for the groups to use and organizing a series of synchronization exercises to ensure consistency among the analyses that were developed in the group. This was an important step that allows straightforward assessment in the final arbitration among the different proposed analyses.

The UVa-ND analysis strives to pursue simple ideas first, graduating to complicated techniques only once they are demonstrated to be necessary. The most crucial distribution in this search is the mass of the WW system, $\mathrm{M}_{\mathrm{lvjj}}$ : if present, signal events will favor values for the WW system mass near the true mass of the Higgs; whereas for the largest background, production of a single $\mathrm{W}$ boson with hadronic jets, the WW system mass will not favor any single value. The separation of the signal peak from the background continuum is another virtue of this channel, since unlike the $\mathrm{H} \rightarrow$ $\mathrm{WW} \rightarrow$ lvlv mode the WW system mass is nearly fully reconstructible given the presence of just one neutrino. We found that the shape predictions from Monte Carlo were reasonable; hence our initial plan in this analysis was to use $\mathrm{MC}$-derived shapes for $\mathrm{M}_{1 \mathrm{vjj}}$, 
comparing the total prediction for the backgrounds and signal to what is observed in data to derive an upper limit on the production cross section of the Higgs in this channel. Although the MC-derived shapes appear reasonable in calibration sample studies, there were indications that the background normalization needed a more authentic determination. To this end, Boutle developed a technique using template fits to data events outside our signal region to determine new normalizations for the $\mathrm{W}+\mathrm{jets}$ and QCD backgrounds.

Using simply the improved normalizations for the backgrounds from the sideband fits and MC-derived shapes for all processes, one can achieve an a priori limit (assuming $2 \mathrm{fb}^{-1}$ of CMS data and considering the combined analysis from the electron and muon channels, as in Fig. 4) that is approaching standard model sensitivity, reaching $\mathrm{x} 2$ the standard model prediction for $\mathrm{M}_{\mathrm{H}}=400 \mathrm{GeV} / \mathrm{c}^{2}$. Further refinements to the technique are in progress, particularly a data-derived $\mathrm{M}_{\mathrm{lvjj}}$ shape for the dominant $\mathrm{W}+\mathrm{jets}$ background. Early indications are promising; with the envisioned refinements and the inclusion of the complete 2011 data sample, this result should play a powerful role in the CMS Higgs program. We anticipate achieving an approved result by the beginning of CY 2012 and that a paper from this result will be available in early 2012.

Fig 4. A priori limit as a function of Higgs mass assuming $2 \mathrm{fb}^{-1}$ of CMS 2011 data and SM Higgs production. $\mathrm{H} \rightarrow \mathrm{WW} \rightarrow \mathrm{e} / \mathrm{mu} \mathrm{v}$ jj channels are combined. 


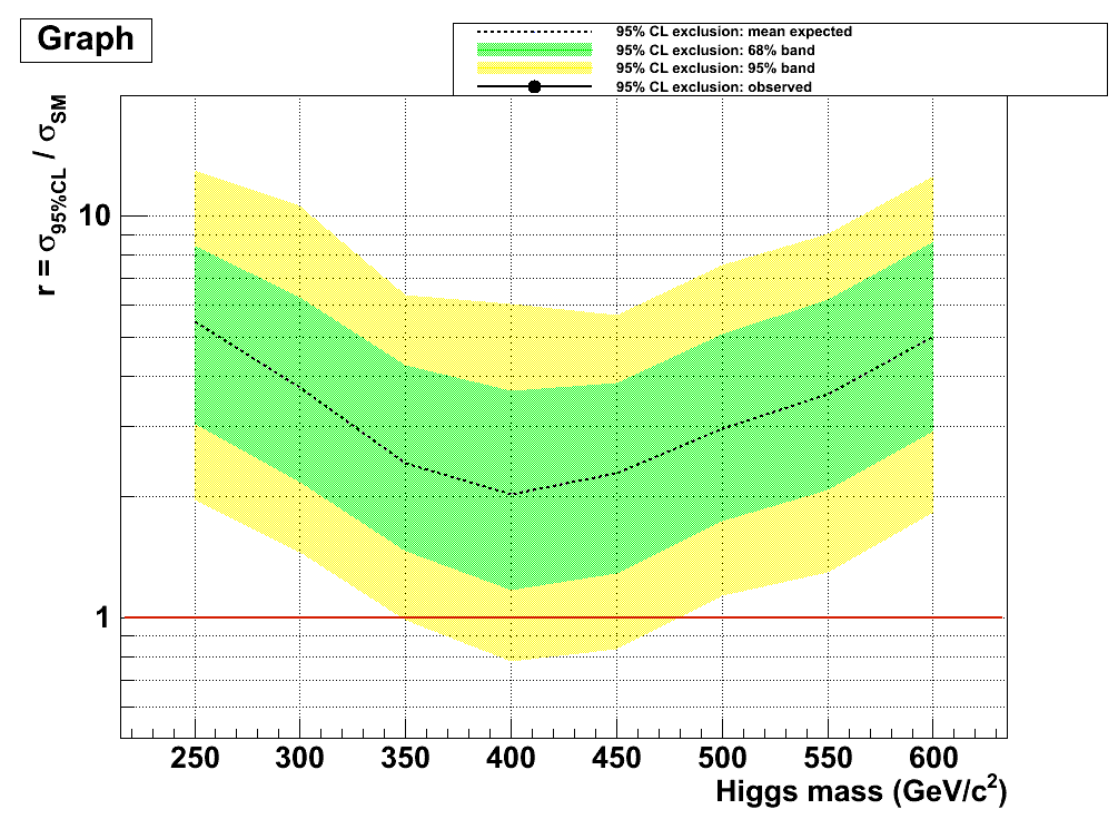

\section{IVd. A Measurement of the Efficiency for Tagging b-jets at CMS (Neu):}

An additional significant contribution to the CMS physics program came in the area of b-tag algorithm deployment. Neu performed in early 2010 the first in-situ calibration of the efficiency of the CMS b-tagging algorithms. Given the unproven nature of the CMS detector simulation's treatment of high energy collisions, this datadriven calibration was essential for utilizing the tagging algorithms that were used in early high $\mathrm{P}_{\mathrm{T}} \mathrm{b}$-jet based analyses. The technique was first deployed in the data from the spring 2010 LHC run, and it exploits the unique characteristics of semileptonic decays of $\mathrm{B}$ hadrons to extract the efficiency for lifetime-tagging jets from $\mathrm{b}$ quark production. A physics analysis in its own right, the results of this effort are made available to the entire collaboration, enabling analyses in the CMS TOP, QCD, EWK and SUSY PAGs. Neu supervised a UCSB student in this effort in 2010.

We expect to get involved in data-driven b-tag efficiency measurements again in 2012, this time using the ttbar sample to provide a data-driven efficiency for tagging $b$, charm and light flavor jets. This is especially interesting to Neu given his long history in b-tag algorithm development.

\section{IVe. An analysis of photon + jets (Lin, and Hirosky):}

The measure of prompt photon production provides a direct experimental probe 
into hard scattering dynamics, serves as a testing ground for perturbative QCD, and constrains possible background for important new physics searches. Quark-gluon Compton scattering producing a high $\mathrm{pT}$ photon also provides information on the proton gluon distribution, which is otherwise only indirectly constrained by the analysis of scaling violations in deep-inelastic scattering e-p collisions. Because of the dominance of the Compton-like process in the LHC data, these are expected to provide new constraints on the low-x gluon density in the proton[1]. We are preparing the first CMS measurement of the triple-differential di-photon plus jet cross section in bins of pT and rapidity. This work is in collaboration primarily with postdoc V. Chetluru (Fermilab) and involvement of D. Mekterovic (postdoc National Central Univ.,Chung-Li) and students from Ankara and Delhi. Among the responsibilities of the UVa group is the derivation of the dominant corrections and systematics affecting the quality of the analysis arising from the determination of photon purity. We have contributed to general studies in the QCD photon group to evaluate systematics related to the non-perturbative model dependencies in photon identification from underlying event and fragmentation models and Lin serves as the representative of the QCD photon group for the generation of necessary MC samples used in these analyses.

Related to this measurement we have also developed a high-level trigger (HLT) to collect data with significantly higher background rejection for a future photon plus heavy flavor and new physics search analyses. Without such trigger innovations, these analysis channels are effectively excluded. Our trigger was incorporated into the CMS HLT suite this summer. In addition to searching for anomalous production of certain heavy flavor final states, photon plus heavy flavor production is sensitive to the charm and gluon densities in the proton (and any unexpected contribution from bottom in the new regions of parton-x to be probed with LHC data). These data can provide additional constraints on poorly understood aspects of parton distribution functions and fragmentation models and thus improve modeling for poorly constrained $\mathrm{X}+$ heavy flavor production processes to better model backgrounds to new physics, such as associated production of a low-mass Higgs boson where important heavy flavor backgrounds suffer large normalization uncertainties. We are also intending to extend the measurement of the triple differential cross section to perform model independent and targeted searches in photon plus jet final 
states. These states are applicable to searches for more exotic physics such as excited quarks, unexpected dynamics affecting angular distributions, certain GMSB (and other) SUSY searches including heavy flavor (with the addition of missing $\mathrm{E}_{\mathrm{T}}$ ), and because electrons are not vetoed in the trigger selection, may further contribute to $3^{\text {rd }}$ generation leptoquarks with $\tau \rightarrow \mathrm{e}$. We will pursue a active research program that focuses first on high quality standard model measurements and extending the interpretation of these measurements to enhance sensitivities to the new physics signatures above.

\section{UVa KTeV Physics Activities}

At present, 50 papers (mostly PRL with an admixture of PRD) have been published by $\mathrm{KTeV}$. We have been either the primary authors or contributed in a significant way to a significant number $(\sim 1 / 4)$ of the analyses leading to the papers or as godparents. We have been responsible for what was, for an extended period of time, the second most cited work in $\mathrm{KTeV}$, the first discovery of a CP violating effect in a dynamic angular distribution (see PRL 96, 101801). Five UVa students have received PhDs from our participation in $\mathrm{KTeV}$.

There is still a $\mathrm{KTeV}$ analysis effort in progress:

a) At this time, Cox with a former student Michael Ronquest is working on the determination of the differences in $\eta_{+-}$and $\eta_{+--.}$This analysis differs from previous analyses in as much as we are determining simultaneously all the amplitudes necessary to determine $\eta_{+-}-\eta_{+-}$from one set of data in one analysis.

b) Cox with another former student, David Phillips recently completed the search for a light particle using the mode $\mathbf{K}_{\mathbf{L}} \rightarrow \boldsymbol{\pi}^{+} \boldsymbol{\pi}^{-} \boldsymbol{\mu} \boldsymbol{\mu}$ where the $\mu \mu$ final state would originate from the decay of an $\mathrm{H}$ particle produced in the decay $\mathbf{K}_{\mathbf{L}} \rightarrow \boldsymbol{\pi}^{+} \boldsymbol{\pi}^{-} \mathbf{H}$. The analysis reached branching ratio upper limits of $10^{-10}-10^{-11}$ in rejecting various theoretical hypotheses and reporting non-observation of such a particle. The final paper has been just published in Phys. Rev. Letters 107.201803. 


\title{
Final Close-Out Report: University of Virginia Task C
} FY 1997-2012

\author{
Prepared by E. Craig Dukes \\ Report Submitted August 8, 2012 \\ for \\ Department of Energy Award No.: DE-FG02-97ER41027
}




\section{Summary}

The Task C group received its first funding from the DOE in 1997, with the award, DE-FG0297ER41027, which was in effect until May, 2012. The group was initiated and has been lead by E. Craig Dukes the entire period. This document summarizes the work that has been done in this period by the Task $\mathrm{C}$ group, with emphasis on the work done is the last three-year funding period. For a list of publications see the Fermilab HyperCP, D $\varnothing$, and MIPP websites, which we do not reproduce here for brevity. $\mathrm{CKM}, \mathrm{NO} \nu \mathrm{A}$, and Mu2e have only published technical documents to date.

\section{HyperCP: $1997-2006$}

Project Personnel: Faculty: S. Conetti, E.C. Dukes, K. Nelson; Postdocs: C. Durandet; Graduate Students: M. Huang, T. Holmstrom, C. Materniak; Undergraduate Students: M. Demming.

The HyperCP experiment was designed to search for rare phenomena in the decays of chargedstrange particles, in particular $C P$ violation in $\Xi$ and $\Lambda$ hyperon decays, with a sensitivity far beyond previous limits. At the time the experiment was proposed and run, $C P$ violation had only been seen in the kaon system, direct $C P$ violation had never been seen, and $C P$ violation had never been observed in any baryon decay. Observation in hyperons would have established all three phenomena. Theoretical predictions suggested that standard-model $C P$ violation was within reach of HyperCP. The Task $\mathrm{C}$ group initiated the experiment with Berkeley, and Dukes served as its co-spokesperson (with Kam-Biu Luk) throughout its duration. A new high-rate spectrometer was fabricated and the experiment took data in two runs: 1997 and 1999.

The Virginia Task C group played a major role in the fabrication of the spectrometer. Our responsibilities included:

- the design and fabrication of the four upstream high-rate MWPC's,

- the design and fabrication of the amplifiers for all the MWPC's,

- the design and fabrication of the hadronic calorimeter,

- the design and fabrication of the OS hodoscope (for the 1999 run),

- the design and fabrication of the upgraded SS hodoscope stand (for the 1999 run),

- the design and implementation of the trigger,

- the development of the processing farm (with LBNL), and

- the running of the Fermilab analysis farm.

The Task $\mathrm{C}$ group played a major role in the reconstruction of the data and the analysis of the 231 billion events, the largest number ever taken by a particle physics experiment at the time. The Virginia Task C group, with LBNL, was responsible for setting up and running the raw event processing, an enormous task as some 30,000 magnetic tapes were analyzed, and a total of $35,847,274$ histograms with information on reconstruction and detector performances were generated! At the International Conference on Computing in High Energy and Nuclear Physics the conference summary speaker called this work a "Tour de Force — by a small experiment" and said that it was "Rather Amazing — processed 240 billion events in 11 months!"

The Virginia group was responsible for writing or co-writing roughly half of the 14 HyperCP publications, and members served as godfathers on almost every other publication. All of the Hyper $C P C P$-violation analysis were carried out by the Task $\mathrm{C}$ group, including the raison d'être for the experiment: the search for $C P$ violation on $\Xi^{-} / \Xi^{+}$decays. Some highlights from these analyses include the following. 
- The most sensitive search for $C P$ violation in hyperon decays, with a twenty times smaller error than that of the previous best result, and the first such result that constrains some SUSY predictions of an effect.

- First observation of the FCNC $K^{-} \rightarrow \pi^{-} \mu^{+} \mu^{-}$and resolution of a discrepancy in two BNL measurements of the $K^{+} \rightarrow \pi^{+} \mu^{+} \mu^{-}$branching ratio.

- First reported high-statistics, high-resolution search for pentaquark, and one of the first null results.

- First evidence of parity violation in $\Omega^{-}$decays, with the measurement of a nonzero $\alpha$ decay parameter, currently the most precisely measured hyperon $\alpha$ parameter.

- First precision test of $C P$ violation in $\Omega^{-}$decays.

- Observation of the rarest baryon decay ever, $\Sigma^{+} \rightarrow p \mu^{+} \mu^{-}$. Evidence suggests that the decay proceeds via an hitherto unknown intermediate state, consistent with the sgoldstino.

- First precision measurement of the $\beta$ parameter in $\Xi^{-} \rightarrow \Lambda \pi^{-}$decays, suggesting that it is larger than chiral-perturbation theory predictions. It is the most precisely known $\beta$ parameter.

- Searches for forbidden hyperon decays that go orders of magnitude in sensitivity beyond previous results.

We did not succeed in seeing $C P$-violation in the hyperon system, at a sensitivity of $0.38 \times 10^{-4}$.

\section{CKM Experiment: 1999-2004}

Project Personnel: Faculty: E.C. Dukes, K. Nelson; Postdocs: L. Lu, R. Godang, G. Niculescu; Graduate Students: none; Undergraduate Students: D. Algoso, J. Gannon, L. Nguyen, C. Noe, F. Ross, H. Zaidi.

The Task C group was a charter member of the Fermilab CKM (E905) collaboration, beginning in the summer of 1999. The goal of the experiment was to test the hypothesis that a single phase in the CKM matrix is the sole source of $C P$ violation, through a $10 \%$ measurement of the CKM matrix element $V_{t d}$, via the decay $K^{+} \rightarrow \pi^{+} \nu \bar{\nu}$. The experiment was approved in June, 2001 and was expected to "form the core of a $120 \mathrm{GeV}$ fixed-target program," for a future fixed target program at the Fermilab Main Injector. ${ }^{1}$ Data taking was expected to begin in 2007-2008.

The Virginia Task C group had significant hardware, software, and administrative responsibilities on CKM. These included:

- The design and construction of the Upstream Magnetic Spectrometer (UMS) and Kaon Entrance Angle Tagger (KEAT).

- The design and construction of the Beam Time Stamp Module (BTSM).

- The design and construction of the front-end electronics.

- The simulation of the UMS and Kaon Entrance Angle Tracker (KEAT).

- Chairing the Experimental Council (Nelson).

We received supplemental funding in 2001 and built and tested prototypes of both the BTSM and UMS/KEAT high-rate wire chambers. We also designed and built prototype preamplifiers for the wire chambers, as well as a prototype two-phase integrator similar to the KTeV QIE for the veto system. We also did extensive simulation work which included: setting up (from scratch) a detailed GEANT-based simulation of the UMS and KEAT, writing a tracking program for both detectors, and various other studies such as the feasibility of a silicon tracker in lieu of wire chambers.

In September, 2004 CKM was cancelled by the newly formed P5 committee due to funding

\footnotetext{
${ }^{1}$ Letter from M. Witherell to CKM, July 6, 1999.
} 
considerations. All work by the Task $\mathrm{C}$ group ended at that time. As a footnote, Fermilab has recently approved a new kaon experiment, ORKA (P1021), which will make a high-precision measurement of $K^{+} \rightarrow \pi^{+} \nu \bar{\nu}$ in a different manner than CKM. We have been asked to join, but existing commitments preclude us doing so.

\section{MIPP Experiment: 2003-2004}

Project Personnel: Faculty: E.C. Dukes, K. Nelson; Postdocs: L. Lu, A. Norman; Graduate Students: C. Materniak; Undergraduate Students: none.

In 2003 the Task C group joined the MIPP collaboration, the goal of which was to explore a variety of phenomena in hadronic particle production, including crucial measurements needed for the next generation of neutrino experiments making high-precision neutrino oscillation and $C P$-violation measurements. (Note that better pion-production measurements are also needed for the Mu2e experiment, as model calculations using FLUKA, GHEISHA, etc. differ by a factor of 6!) To some extent MIPP was an experiment to bridge the gap between the end of HyperCP and the beginning of CKM, and after CKM was cancelled, MECO. The Task C group originally anticipated a limited role in MIPP, given our responsibilities on other higher-priority projects (CKM and then MECO). The running of MIPP, which began in early 2004, consumed far more Task C group resources than originally anticipated, including 2672 hours of shifts, the most of any collaborating institution. The limited manpower resources of the experiment, coupled with the hardware strengths of the group, resulted in the Task $\mathrm{C}$ group assuming a much larger role including the

- refurbishing the drift chambers and their electronics,

- installation, maintenance, and operation of the hadronic calorimeter (built for HyperCP),

- design and installation of the trigger,

- the beam chamber/drift chamber shared electronics,

- the beam Cerenkov high-speed DAQ subsystem,

- design and implementation of the high-voltage control system and monitoring interfaces, and

- the design and fabrication of the fiber crosshair hodoscope.

The Task C group also designed and proposed an upgrade to the TPC front-end electronics that would have increased the readout rate from $60 \mathrm{~Hz}$ to $3,000 \mathrm{~Hz}$.

MIPP commissioning began in February 2004. The responsibility of the Task $\mathrm{C}$ group at that time was the hadron calorimeter and the high-voltage control and monitoring system. In April 2004, difficulties with the commissioning of the tracking chamber subsystems resulted in the Task C group assuming responsibility for the drift chambers and their associated front-end readout systems. The chambers were made operational by the end of June 2004. In May 2004 the Task C group also designed a new particle identification trigger, which was installed and commissioned during June and July 2004. Subsequently, the Task C group designed an interaction trigger using the drift chambers. It was installed and brought online in late July 2004. Following this the Task C group rebuilt the entire trigger, which remained in operation throughout the 2005 production run.

Funding constraints unfortunately reduced our active participation in MIPP at the end of 2004, and all Virginia activities ended in 2005.

\section{DØ Experiment: 2006-2011}

Project Personnel: Faculty: E.C. Dukes, R. Hirosky, K. Nelson; Postdocs: D. Brown, M. Buehler, A. Kryemadhi, A. Norman; Graduate Students: E. Munyangabe, H. Nguyen, S. Zelitch; Under- 
graduate Students: E. Carr, B. Ionescu, D. Kamensky, Y. Yao, E. Xu

Hirosky joined Virginia as a junior faculty member in the fall 2000, working on DØ. In 2006 his grant which was merged into Task C, with him continuing to work on DØ full time. Hirosky's fraction on Task C started at $80 \%$ in 2006, and by 2011 it had ramped down to 10\%, as his fraction on Task B (CMS) correspondingly increased. After MECO was cancelled both Dukes and Nelson joined $\mathrm{D} \varnothing$, while still taking data with MIPP and planning $\mathrm{NO} \nu \mathrm{A}$ and Mu2e. Nelson went to Johns Hopkins on leave in 2006 and subsequently resigned his faculty position at Virginia to take a permanent position at their Applied Physics Laboratory.

Hirosky had major roles in many different aspects of $\mathrm{D} \varnothing$, in particular with the Level-2 trigger, part of which he designed and built. This continues as he now serves as physics coordinator for the collaboration. Highlights of the Task C group's work on DØ include the follow.

- Buehler served as DØ run coordinator in 2008-2009.

- Buehler was the Higgs group Trigger Board representative.

- Buehler was co-head of the Central Track Trigger Group.

- Hirosky was co-head of the Level-2 Trigger Group.

- Hirosky served as chair of the jet energy scale certification board.

- Hirosky served as "Data Commissioner."

- Brown was leader of the QCD group, which Under his direction the group completed an impressive spate of new measurements testing the underlying dynamics of the theory of strong interactions.

- Norman was a convener of the lepton flavor violation subgroup.

- Zelitch and Munyangabe were DAQ expert shifters and Level-2 Experts.

- Buehler, Hirosky, and Zelitch pioneered a search for direct Higgs production in $H \rightarrow W W \rightarrow$ $\mu \bar{\nu} j j+C . C$. , which lead to a significant increase in event yield.

- Mulhearn, Nguyen, and Kamensky worked on the low mass Higgs channel $Z H \rightarrow \ell \ell b \bar{b}$, where they took a leading role unifying the channels in this analysis and developing a full matrix element-based classifier to further improve sensitivity.

- Mulhearn took a leading role in operations of the DØ Level 2 trigger.

\section{NO $\nu$ A Experiment: 2004-2012}

Project Personnel: Faculty: E.C. Dukes, K. Nelson; Postdocs: R. Ehrlich, M. Frank, A. Norman; Graduate Students: Z. Wang; Undergraduate Students: J. Gran, M. Shields, H.Tammaro, K. Langeron, D. Mudd; Technicians/Engineers: S. Goadhouse, B. Mason.

In early 2003 we started working on an off-axis neutrino experiment designed to exploit the NUMI beamline, our longstanding interest in fundamental symmetries of nature interest piqued by the prospects of observing $C P$ violation in neutrino oscillations. Given our CKM responsibilities our initial participation was minimal, however it picked up substantially when CKM was cancelled in 2004, and more so with the cancellation of MECO in late 2005. $\mathrm{NO} \nu \mathrm{A}$ currently is the major thrust in the group's efforts, where we have major fabrication responsibilities.

The $\mathrm{NO} \nu \mathrm{A}$ collaboration is building a 15,000 ton, highly-grained, low-Z, total absorption calorimeter to be placed at an off-axis angle of $14 \mathrm{mrad}$ to an upgraded NuMI neutrino beam. The detector is optimized to observe electron neutrino appearance in a muon neutrino beam. The long baseline allows the possibility of resolving the neutrino mass hierarchy.

The Task $\mathrm{C}$ group has major roles in two major mission-critical subsystems of the $\mathrm{NO} \nu \mathrm{A}$ detector: the Power Distribution System (PDS), which powers all of the NO $\nu$ A electronics, and 
the Detector Controls \& Monitoring Systems (DCS), which is the user's window into controlling $\mathrm{NO} \nu \mathrm{A}$, and monitors, alarms, and logs system-critical parameters. We are solely responsible for the design, prototyping, fabrication, and installation of the PDS, for which Dukes serves as Level 3 manager. The DCS was designed by Norman when he was a member of the Task $\mathrm{C}$ group. In 2009 the NO $\nu$ A project management requested that Task C postdoc Norman be appointed head of the DAQ integration project, as part of a critical reallocation of effort initiated by $\mathrm{NO} \nu \mathrm{A}$ after the Fermilab-led DAQ group had been permanently reassigned to other projects during the 2008 funding hiatus. (A working DAQ was a higher priority than the DCS.) This left the DCS orphaned for about a year after which Chad Johnson of Indiana agreed to take it over for its installation in the Near Detector. In early 2011 Johnson was replaced by Athanasios Hatzikoutelis of Tennessee. However, the DCS remained severely undersubscribed and Virginia postdoc Frank immediately delved into the DCS, serving as the coordinator for the electronics and instrumentation integration, and playing an increasingly important role as Hatzikoutelis, due to visa problems, was not able to be permanently resident at Fermilab. Frank essentially ran the DCS as Level 3 manager, until Hatzikoutelis's visa problem was solved, and then at the request of the $\mathrm{NO} \nu \mathrm{A}$ project management became head of the Block Instrumentation Group.

In April 2011 we hired technician Bridget Mason to help with the fabrication and testing of the Power Distribution System components. She will continue to be paid from $\mathrm{NO} \nu \mathrm{A}$ project funds until the testing is complete in May 2012.

We are receiving $\$ 2,571,318$ in $\mathrm{NO} \nu \mathrm{A}$ project funds for this work.

The Task $\mathrm{C}$ group has made other contributions to $\mathrm{NO} \nu \mathrm{A}$ besides the Power Distribution System and the Detector Control \& Monitoring Systems. We have been an intimate part of the design of the readout infrastructure, and through that the design of the Far Detector building: detector drawings made by Dukes were used for the initial building design. In 2009 we played an integral part of the redesign of the DAQ, with Virginia postdoc leading the effort as Level 3 manager for the DAQ Hardware/Software Integration, which included redesign of the data buffering scheme, data-driven triggering, global trigger design, the data dispatcher, and the data logger. The Task C group also helped with the commissioning of the Data Concentrator Module (DCM), a key component of the DAQ designed by Fermilab. Virginia electrical engineer Goadhouse made several trips to Fermilab, where he provided invaluable service in debugging the DCM, including finding a hardware problem with the boards that prevented them from booting.

Finally, we note that we have responsibilities beyond leading the Power Distribution System and the Block Instrumentation Group. Dukes is a member of the NO $\nu$ A Executive Committee the only non-MINOS member outside of the project manager — an elected body that advises the co-spokespersons on scientific, technical, financial, and organizational policies. Dukes also serves on the $\mathrm{NO} \nu \mathrm{A}$ Speakers Board and is the Institutional Board representative for Virginia. Frank and Wang are both on-call Detector Control \& Monitoring Systems experts.

A list of $\mathrm{NO} \nu \mathrm{A}$ work completed by the Task $\mathrm{C}$ group follows.

- Completed the detailed layout of the Power Distribution System for the Near Detector in its surface building, as well as for the Far Detector.

- Completed the design and tested V3.2 and V3.3 of the Power Distribution Box and its component FEB, DCM, and IND boards.

- Fabricated 15 Power Distribution Boxes for the Near Detector and for test benches at Fermilab, Harvard, and Virginia.

- Designed and set up three test stands for: (1) the Power Distribution Boxes, (2) the highvoltage power supplies, and (3) the low-voltage power supplies.

- Ordered and tested all high- and low-voltage power supplies needed for the Near Detector. 
- Fabricated all of the cable harnesses, high-current cables, high-voltage cables, sense cables, and ground cables needed for the Near Detector.

- Designed and fabricated the high-voltage breakout boxes needed for the Near Detector.

- Designed and fabricated the breaker boxes needed for the Near Detector.

- Installed and successfully commissioned the Power Distribution System for the Near Detector.

- Designed, reviewed, and implemented large portions of the DAQ readout chain, the global triggering schema, data-driven triggering mechanisms, data logging and event building system, event dispatcher system, and preliminary run-control design.

- Help set up the DAQ test stand at Feynman.

- Took over the embedded systems infrastructure for the Data Concentrator Modules.

- Developed the bootloader code for the Data Concentrator Modules and Timing Units.

- Developed the full raw-data format (described in a 43-page long document) as well as software simulation modules to inject raw data into the DAQ chain.

- Completed and supported APD Temperature Monitor for Near Detector operation.

- Established organizational infrastructure for the DCS group.

- Established on-call expert shifts and served as experts for Near Detector commissioning.

- Aided in the testing and evaluation of EPICS software.

- Developed a fast Monte Carlo simulation to perform high-statistic studies of detector acceptance under varying geometries, in particular to study the effect of offsets in plane alignment.

\section{Mu2e Experiment: 2004-2012}

Project Personnel: Faculty: E.C. Dukes, C. Group (not supported on project funds), R. Hirosky, K. Nelson, P.Q. Hung; Postdocs: R. Ehrlich, M. Frank, A. Norman; Y. Oksuzian (not supported on project fund); Graduate Students: none; Undergraduate Students: J. Gran;

After the cancellation of CKM in late 2004 we turned our efforts to the MECO experiment, whose goal is to search for lepton-flavor violation through the process $\mu^{-} N \rightarrow e^{-} N$. and which was being planned to run at Brookhaven National Laboratory as part of the RSVP project. MECO was to serve as our near term project, while $\mathrm{NO} \nu \mathrm{A}$ would be our long-term goal.

The Virginia Task $\mathrm{C}$ group had two responsibilities on MECO:

- the design and fabrication of the front-end electronics for the straw detectors, and most likely for the electromagnetic calorimeter as well, and

- the fabrication of the Cosmic Ray Veto detector.

Unfortunately, MECO was cancelled in the fall of 2005. In early 2006 Dukes and a handful of other physicists began exploring options for mounting the experiment at Fermilab in early 2006. In 2008 Dukes took a sabbatical at Fermilab to work on the $\mathrm{NO} \nu \mathrm{A}$ prototype near detector. $\mathrm{NO} \nu \mathrm{A}$ 's funding was cut the previous December and so Dukes mainly worked on exploring how to mount a MECO-like experiment at Fermilab. This included writing the Mu2e proposal, which was approved in the fall of 2008, and in the fall of 2009 the experiment achieved CD-0 status. The collaboration has blossomed to 28 institutions and 135 physicists and engineers.

The Task $\mathrm{C}$ group work done on Mu2e is itemized below.

- We hold key leadership positions with: (1) Dukes serving as the head of the Mu2e Institutional Board, (2) Dukes leading (as Level 2 manager) the Cosmic Ray Veto (CRV) group (the only Level 2 manager from an academic institution), (3) Group leads the CRV module fabrication effort (as Level 3 manager), which will be done at Virginia, and (4) Oksuzian leads the fiber effort (as Level 3 manager). As part of Dukes's leadership responsibilities he: (1) leads the bi-weekly Cosmic Ray Veto meetings, (2) presents the status of the Cosmic Ray Veto at the 
plenary sessions of the collaboration meetings, and (3) presented the status of the Cosmic Ray Veto at the Director's design review in May, 2011, the Director's review in March, 2012, and the DOE CD-1 review in June, 2012.

- Dukes has lead the design of the Cosmic Ray Veto which was completed for the DOE CD-1 review.

- Both Group and Oksuzian have led the Fermilab-based R\&D effort that measured the efficiencies with an early prototype module in a cosmic-ray test stand set up in the CDF assembly hall, as well as in the Meson Test Beam.

- Ehrlich is in charge of the Mu2e event display package and the cosmic-ray background simulation effort.

- Frank is helping with the vital neutron background simulations, and will eventually take over that work.

- Finally, Virginia electrical engineer, Goadhouse, is responsible for the Readout Controller that takes data from the front-end boards and sends it to the DAQ.

Note that neither Hirosky nor Hung receive any support from Task $\mathrm{C}$ funds for their Mu2e efforts, which are minimal at this time. Additional non-Task C Mu2e members include two faculty members (Paschke and Počanić) and senior research scientist Frlez from the Virginia nuclear physics group. Presently their Mu2e research effort is minimal as they are consumed by other ongoing activities, but we expect in several years they will be able to ramp up their MuRe involvement. 